\title{
PCK2 opposes mitochondrial respiration and maintains the redox balance in starved lung cancer cells
}

\author{
Gabriele Bluemel $^{\mathrm{a}}$, Mélanie Planque ${ }^{\mathrm{b}, \mathrm{c}}$, Corina T. Madreiter-Sokolowski ${ }^{\mathrm{d}, \mathrm{e}}$, \\ Theresa Haitzmann $^{\mathrm{a}}$, Andelko Hrzenjak ${ }^{\mathrm{a}, \mathrm{f}}$, Wolfgang F. Graier ${ }^{\mathrm{d}, \mathrm{g}}$, Sarah-Maria Fendt ${ }^{\mathrm{b}, \mathrm{c}}$, \\ Horst Olschewski ${ }^{\text {a,f }}$, Katharina Leithner ${ }^{\text {a, }}$,"* \\ ${ }^{a}$ Division of Pulmonology, Department of Internal Medicine, Medical University of Graz, Graz, Austria \\ ${ }^{\mathrm{b}}$ Laboratory of Cellular Metabolism and Metabolic Regulation, VIB-KU Leuven Center for Cancer Biology, VIB, Leuven, Belgium \\ ${ }^{\mathrm{c}}$ Laboratory of Cellular Metabolism and Metabolic Regulation, Department of Oncology, KU Leuven and Leuven Cancer Institute (LKI), Leuven, Belgium \\ ${ }^{\mathrm{d}}$ Gottfried Schatz Research Center for Cell Signaling, Metabolism and Aging, Molecular Biology and Biochemistry, Medical University of Graz, Graz, Austria \\ ${ }^{\mathrm{e}}$ Energy Metabolism Laboratory, Institute of Translational Medicine, Department of Health Sciences and Technology, Swiss Federal Institute of Technology (ETH) Zurich, \\ Zurich, Switzerland \\ ${ }^{\mathrm{f}}$ Ludwig Boltzmann Institute for Lung Vascular Research, Graz, Austria \\ ${ }^{g}$ BioTechMed-Graz, Graz, Austria
}

\section{A R T I C L E I N F O}

\section{Keywords:}

Cancer metabolism

Gluconeogenesis

Adaptation

Metabolic flexibility

Mitochondria

Respiration

Redox balance

\begin{abstract}
A B S T R A C T
Cancer cells frequently lack nutrients like glucose, due to insufficient vascular networks. Mitochondrial phosphoenolpyruvate carboxykinase, PCK2, has recently been found to mediate partial gluconeogenesis and hence anabolic metabolism in glucose starved cancer cells. Here we show that PCK2 acts as a regulator of mitochondrial respiration and maintains the redox balance in nutrient-deprived human lung cancer cells. PCK2 silencing increased the abundance and interconversion of tricarboxylic acid (TCA) cycle intermediates, augmented mitochondrial respiration and enhanced glutathione oxidation under glucose and serum starvation, in a PCK2 reexpression reversible manner. Moreover, enhancing the TCA cycle by PCK2 inhibition severely reduced colony formation of lung cancer cells under starvation. As a conclusion, PCK2 contributes to maintaining a reduced glutathione pool in starved cancer cells besides mediating the biosynthesis of gluconeogenic/glycolytic intermediates. The study sheds light on adaptive responses in cancer cells to nutrient deprivation and shows that PCK2 confers protection against respiration-induced oxidative stress.
\end{abstract}

\section{Introduction}

Cancer cells undergo metabolic reprogramming for fast growth and proliferation. They utilize large amounts of glucose for the biosynthesis of cellular building blocks [1,2]. Moreover, certain amino acids such as glutamine are consumed at high rates in order to support anabolic metabolism [1-3]. Despite the induction of angiogenesis at an early stage of tumor growth, the nutrient supply is often not sufficient and steep nutrient gradients occur with increasing distance from the vessels [4]. In a murine pancreatic cancer model, glucose levels were substantially lower in the tumor's interstitial fluid than in the plasma, while glutamine levels were similar [5]. Thus, cancer cells need to adapt to a highly variable nutrient supply and starvation conditions $[6,7]$.

As part of their metabolic rewiring, cancer cells are known to limit the complete catabolism of glucose, its entry into the TCA cycle and the rate of mitochondrial respiration by reducing the rate of acetyl-CoA formation at the step of pyruvate dehydrogenase (PDH) [8]. This key metabolic enzyme is repressed by pyruvate dehydrogenase kinase 1 (PDK1), which is induced by oncogenes like c-myc or $\beta$-catenin [9], but also tightly regulated by allosteric inhibition by ATP and NADH [8]. In addition to PDH inhibition other regulatory mechanisms exist to balance TCA cycle activity and mitochondrial respiration. Acetyl-CoA is condensed with oxaloacetate (OAA) in the TCA cycle to form citrate, which eventually results in complete oxidation of acetyl-CoA to two $\mathrm{CO}_{2}$ molecules and subsequent regeneration of OAA. The concentration of OAA and acetyl-CoA are important regulators of the TCA cycle which fuels mitochondrial respiration by its production of reducing equivalents $[8,10]$. In some conditions, including the high metabolic activity of

\footnotetext{
* Corresponding author. Auenbruggerplatz 15, A-8036, Graz, Austria.

E-mail address: katharina.leithner@medunigraz.at (K. Leithner).
} 
cancer cells, TCA cycle intermediates get depleted due to their use in anabolic biosynthetic reactions (cataplerosis) and must be replenished e. g. from glutamate to maintain the function of the TCA cycle [11]. While the factors regulating the TCA cycle and mitochondrial respiration in cancer cells under nutrient replete conditions have been well characterized in the past years [9], their regulation under nutrient limitation is still poorly understood.

Glucose deprivation increases mitochondrial respiration in many cancer cell lines [12]. However, mitochondrial respiration might enhance the formation of potentially damaging reactive oxygen species (ROS) as a certain amount of oxygen consumed by mitochondria is transformed to superoxide anion $\left(\mathrm{O}_{2}{ }^{-}\right)$, [8]. Superoxide is rapidly reduced to $\mathrm{H}_{2} \mathrm{O}_{2}$ by mitochondrial superoxide dismutase. $\mathrm{H}_{2} \mathrm{O}_{2}$ is a signaling molecule, but when produced in excess it can give rise to the extremely reactive and cell damaging hydroxyl radical $(\cdot \mathrm{OH})$ upon reaction with metal cations $\left(\mathrm{Fe}^{2+}\right.$ or $\mathrm{Cu}^{+}$). Thus, $\mathrm{H}_{2} \mathrm{O}_{2}$, is constantly converted to water by catalase, peroxiredoxins or by glutathione (GSH) -dependent enzymes including glutathione peroxidase 1 (GPX1), thereby oxidizing GSH to glutathione disulfide (GSSG) [13]. GSSG is reduced again by glutathione reductase (GSR) using electrons from NADPH [13]. The redox-modulated transcription factor nuclear factor erythroid 2-related factor 2 (NRF2, encoded by NFE2L2) promotes antioxidant responses, among others by up-regulation of GSH synthesis via enhanced cystine import [14,15].

Phosphoenolpyruvate carboxykinase (PEPCK), a key enzyme of gluconeogenesis, catalyzes the GTP-dependent conversion of OAA to the glycolytic intermediate phosphoenolpyruvate (PEP). The enzyme exists in two different isoforms, the cytoplasmic isoform PCK1 (PEPCK-C) and the mitochondrial isoform PCK2 (PEPCK-M). Contrary to the previously assumed restriction to classic gluconeogenic tissues, PCK2 is also expressed in a variety of cancers, including lung cancer, breast cancer and prostate cancer [16-24]. However, PCK2 is also expressed in different non-neoplastic tissues, including the lung [25,26]. The enzyme allows cancer cells to generate glycolytic intermediates from small non-carbohydrate molecules such as glutamine or lactate (reviewed in [27]). We and others showed that PCK2, the prime isoform expressed in lung cancer, promotes the survival and proliferation of lung cancer cells under conditions of low glucose, as well as xenograft growth in vivo [16, $18,19,28]$. In case of low glucose availability, PCK2 mediates the biosynthesis of serine, glycine and purine nucleotides [18,28,29], or the glycerol backbone of phospholipids [19] in cancer cells. It remains unknown, however, whether PCK2 regulates mitochondrial respiration in cancer cells under nutrient starvation. Here we show that PCK2 diminishes the levels of TCA cycle intermediates in nutrient deprived lung cancer cells, thereby suppressing starvation-induced oxygen consumption. Moreover, we reveal that the cataplerotic activity of PCK2 protects lung cancer cells from oxidative stress.

\section{Materials and methods}

Cell lines. The human NSCLC cell line A549 was obtained from Cell Lines Service (Eppelheim, Germany). A549 cells were cultured in DMEM/F-12 (Gibco, Waltham, MA, USA) supplemented with $10 \%$ fetal calf serum (FCS, Biowest, Nuaillé, France) and antibiotics (Gibco). The human NSCLC cell line NCI-H23 (H23) was purchased from American Type Culture Collection (ATCC, Manassas, VA, USA). H23 cells were cultured in RPMI 1640 (Gibco) supplemented with 10\% FCS (Biowest) and antibiotics (Gibco). If not stated differently, cells were plated for all experiments with a density of 22,000 cells per $\mathrm{cm}^{2}$ in normal growth media. After 20-24 h cells were washed twice with phosphate buffered saline (PBS) and treated either with non-starvation or with starvation media. For starvation/non-starvation treatment, glucose and glutamine free DMEM (Gibco) or glucose and glutamine free RPMI SILAC medium (Gibco) were supplemented with $2 \mathrm{mM}$ glutamine, $0.2 / 10 \mathrm{mM}$ (starvation/non-starvation) glucose, $0 \% / 10 \%$ dialyzed FCS and antibiotics. RPMI SILAC was additionally supplemented with $1.15 \mathrm{mM}$ arginine and
$0.27 \mathrm{mM}$ lysine. Cell line authentication was done for both cell lines by Short Tandem Repeat (STR) analysis using the PowerPlex 16HS System (Promega, Madison, WI).

Stable expression of PCK2 shRNA. H23 cells were stably transfected with PCK2 shRNA or non-silencing control shRNA (Qiagen, Hilden, Germany). Puromycin-selection and generation of monoclonal subcultures was performed as described [19]. Maintenance media was supplemented with $0.5 \mu \mathrm{g} / \mu \mathrm{L}$ puromycin (Sigma Aldrich, Waltham, MA, USA) which was omitted during experiments. To confirm the specificity of PCK2 silencing effects, we transfected PCK2 silenced cells with a shRNA resistant PCK2 cDNA construct. Three codons of the shRNA binding site were point mutated without altering the amino acid sequence (Eurofins Genomics, Ebersberg, Germany) and cloned into a pCMV6-AC expression vector (Origene, Rockville, MD); the empty expression vector was used as a control. Cells were transfected with 0.5-1 $\mu \mathrm{g}$ DNA/200,000 cells $48 \mathrm{~h}$ before treatment start with jetPRIME (Polyplus, Illkirch, France) transfection reagent.

PCK2 silencing with siRNA. A549 and H23 cells were transfected either with non-silencing (ctrl) siRNA (Non-targeting pool, Dharmacon, Horizon, Colorado, USA) or with PCK2 siRNA1 (Smart pool PCK2, Dharmacon). PCK2 siRNA2 was a custom designed pool with the sequences GGAUGAGGUUUGACAGUGA and UGGCUACAAUCCAGAGUAA (Dharmacon).

PCK2 overexpression. PCK2 re-expression was performed by transfecting cells with a PCK2 wild-type plasmid (Origene, Rockville, MD); the empty pCMV6-AC expression vector was used as a control. Cells were transfected with $1 \mu \mathrm{g}$ DNA/200,000 cells $48 \mathrm{~h}$ before the start of the treatment.

Stable isotopic tracing. For analysis of TCA cycle metabolite abundance and flux, ${ }^{13} \mathrm{C}_{5}$-glutamine was utilized as a tracer. Cells were treated for $24 \mathrm{~h}$ with glutamine free non-starvation/starvation media supplemented with $2 \mathrm{mM}{ }^{13} \mathrm{C}_{5}$-glutamine. Then cells were washed with saline and metabolism was quenched by immediately freezing the cells on liquid nitrogen.

Sample extraction and gas chromatography-mass spectrometry (GC-MS) and liquid chromatography-mass spectrometry (LC-MS) measurements. Metabolite extraction, derivatization and analysis were performed as described [30]. Metabolites were extracted on dry ice/ice with cold $62.5 \%$ methanol in water, containing norvaline and glutarate as internal standards. Cells were scraped and ice cold chloroform was added. The samples were vortexed at $4^{\circ} \mathrm{C}$ or sonicated to extract metabolites, centrifuged for $10 \mathrm{~min}$ at $4^{\circ} \mathrm{C}$ and the phases were separated. For measurements of polar metabolites, the methanol in water phase was dried by vacuum centrifugation. For GC-MS, samples were derivatized with $20 \mathrm{mg} / \mathrm{mL}$ methoxyamine (Sigma) in pyridine (Sigma) for $90 / 60 \mathrm{~min}$ at $37^{\circ} \mathrm{C}$ and thereafter with $\mathrm{N}$-(tert-butyldimethylsilyl)- $N$-methyl-trifluoroacetamide (TBDMS, Sigma) for $60 / 30 \mathrm{~min}$ at $60^{\circ} \mathrm{C}$.

Separation was performed with an Agilent 7890A GC system coupled to an Agilent 5975C Inert MS system, or an Agilent 7890B GC system coupled to an Agilent 5977A Inert MS system. A DB35MS column was used and helium was used as a carrier gas at a flow rate of $1 \mathrm{~mL} / \mathrm{min}$. A volume of $1 \mu \mathrm{L}$ sample was injected in splitless mode with an inlet temperature of $270^{\circ} \mathrm{C}$. The GC oven was kept at $100^{\circ} \mathrm{C}$ for $1 \mathrm{~min}$, increased up to $105^{\circ} \mathrm{C}$ with a gradient of $2.5^{\circ} \mathrm{C} / \mathrm{min}$, then ramped to $240^{\circ} \mathrm{C}$ with a gradient of $3.5^{\circ} \mathrm{C} / \mathrm{min}$, and after that ramped up to $320^{\circ} \mathrm{C}$ with a gradient of $2.5^{\circ} \mathrm{C} / \mathrm{min}$, which was followed by $4 \mathrm{~min}$ at $320^{\circ} \mathrm{C}$ or kept at $100^{\circ} \mathrm{C}$ for $3 \mathrm{~min}$ and ramped to $300^{\circ} \mathrm{C}$ with a gradient of $3.5^{\circ} \mathrm{C}$ / min. Mass spectrometry was performed at $70 \mathrm{eV}$ and a mass range of 100-650 atomic mass units was measured. Isotopologue distributions of the metabolites were quantified, normalized to protein and corrected for natural abundance with a MATLAB Script (MA, USA) or with IsoCor [31].

GSH, GSSG, NADPH and NADP ${ }^{+}$total abundances were analyzed by LC-MS/MS. Metabolites were extracted as described for GC-MS experiments. Then, vacuum dried samples were resuspended in $80 \%$ methanol 
in water. A Dionex UltiMate 3000 LC System (Thermo Scientific) with a HILIC column (iHILIC®-Fusion HILIC Column, SS, $150 \times 2.1 \mathrm{~mm}, 1.8$ $\mu \mathrm{m}, 100 \AA$ ) at $28^{\circ} \mathrm{C}$ and a flow rate of $0.2 \mathrm{~mL} / \mathrm{min}$ was utilized for separation. A gradient was applied for $38 \mathrm{~min}$ (solvent A: acetonitrile, solvent B: $10 \mathrm{mM}$ ammonium acetate, $\mathrm{pH}$ 9.3) (0 min: $8 \% \mathrm{~B}, 2 \mathrm{~min}: 8 \%$ B, 7 min: 35\% B, 16 min: 42\% B, 26 min: 75\% B, 28 min: 75\% B, 30 min: $8 \%$ B, 38 min: $8 \%$ B). The MS operated in negative mode with a spray voltage of $2.5 \mathrm{kV}$, capillary temperature was $350^{\circ} \mathrm{C}$, sheath gas at 50 and auxiliary gas at 10 . At least two transitions per metabolite were measured to ensure the specificity of the method, the following transition (precursor ion $>$ product ion; collision energy) were used for the quantification: GSH (306 > 143; 20V), GSSG (611 > 306; 24V), NADPH $(744>408 ; 40 \mathrm{~V}), \mathrm{NADP}^{+}(742>273 ; 40 \mathrm{~V})$. Data were collected and analyzed using the Xcalibur software (Thermo Scientific).

GSH isotopologues were detected by using LC-MS as previously described [32]. Sample preparation occurred as previously stated for GC-MS measurements. A Dionex UltiMate 3000 LC System (Thermo Scientific) was coupled to a Q-Exactive Orbitrap mass spectrometer (Thermo Scientific). Samples were resuspended in $\mathrm{H}_{2} \mathrm{O}$. Separation was achieved with a flow rate of $0.25 \mathrm{~mL} / \mathrm{min}$, by utilizing a $\mathrm{C} 18$ column (Acquity UPLC HSS T3 $1.8 \mu \mathrm{m} 2.1 \times 100 \mathrm{~mm}$ ) at $40^{\circ} \mathrm{C}$ and a gradient for 40 min (solvent A: $\mathrm{H}_{2} \mathrm{O}, 10 \mathrm{mM}$ tributyl-amine, $15 \mathrm{mM}$ acetic acid solvent B:methanol) (0 min: 0\% B, 2 min: $0 \% \mathrm{~B}, 7 \mathrm{~min}: 37 \% \mathrm{~B}, 14 \mathrm{~min}$ : 41\% B, 26 min: 100\% B, 30 min: 100\% B, 31 min: 0\% B; 40 min: 0\% B). The MS operated in negative full scan mode ( $\mathrm{m} / \mathrm{z}$ range: $70-900)$ using a spray voltage of $4.9 \mathrm{kV}$, capillary temperature of $320^{\circ} \mathrm{C}$, sheath gas at 50 and auxiliary gas at 10 . Data were collected and analyzed using the Xcalibur software (Thermo Scientific).

Seahorse measurements. Cells were plated on Cell-Tak ${ }^{\mathrm{TM}}$ coated XF96 polystyrene cell culture microplates (Seahorse Bioscience, Agilent; Santa Clara, CA, USA) at a density of 40,000 cells per well $24 \mathrm{~h}$ before treatment. Thereafter, cells were cultured for $24 \mathrm{~h}$ in starvation or nonstarvation media. As respiratory substrate we added either $1 \mathrm{mM}$ pyruvate (Sigma) (experiment with H23 ctrl sh_v/PCK2sh_v and PCK2sh_mt cells) or $10 \mathrm{mM}$ lactate (Sigma) (experiments with H23 ctrl sh/PCK2 sh and A549 cells), if not indicated differently. OCR was measured with a XF96 Extracellular Flux analyzer (Seahorse Bioscience). Measurements were performed under basal conditions and after the addition of $2 \mu \mathrm{M}$ oligomycin, $0.5 \mu \mathrm{M}$ (A549)/0.2 $\mu \mathrm{M}$ (H23) carbonyl cyanide-4-(trifluoromethoxy)phenylhydrazone (FCCP) and $10 \mu \mathrm{M}$ antimycin. If indicated, $40 \mu \mathrm{M}$ etomoxir was added (Sigma). OCR was normalized to protein content.

Mitotracker analysis with flow cytometry. Cells were treated for $24 \mathrm{~h}$, washed with PBS and then incubated with $100 \mathrm{nM}$ Mitotracker Green (Thermo Scientific, Waltham, MA, USA) in serum-free media for $30 \mathrm{~min}$. Thereafter, cells were washed with PBS and measured with flow cytometry (CytoFlex, BeckmanCoulter, Krefeld, Germany) in starvation media. Cells were gated for live and single cells.

Confocal microscopy of mitochondria. $2 \times 10^{4}$ cells were plated onto 8-well chamber slides with glass bottom (Ibidi, Gräfelfing, Germany) and treated for $24 \mathrm{~h}$ with non-starvation or starvation media, washed with PBS and incubated with $100 \mathrm{nM}$ Mitotracker Green (Thermo Scientific) in RPMI SILAC supplemented with arginine and lysine for $30 \mathrm{~min}$. Thereafter, cells were washed with PBS and reincubated in the respective treatment medium. Immediately, images of representative cells were taken by Nikon A1 confocal microscope (Vienna, Austria). Images were analyzed by ImageJ (National Institutes of Health (NIH)) with the MiNa plugin [33].

Detection of ROS. For detection of intracellular mitochondrial superoxide, cells were plated and treated after $24 \mathrm{~h}$ with non-starvation or starvation media. Then cells were washed with PBS, trypsinized and incubated with $2.5 \mu \mathrm{M}$ of MitoSox (Thermo Scientific) for $20 \mathrm{~min}$ at $37^{\circ} \mathrm{C}$. After washing with PBS, cells were resuspended in PBS and analyzed by flow cytometry (CytoFlex, BeckmanCoulter). To determine general ROS, cells were treated as described above and ROS were determined with $10 \mu \mathrm{M}$ of CM-H2DCFDA (Thermo Scientific) upon incubation for $30 \mathrm{~min}$ at $37^{\circ} \mathrm{C}$ followed by flow cytometry. Lipid peroxidation was measured in cells treated with or without RSL3 (Selleck chemicals, Houston, TX, USA) with $2 \mu \mathrm{M}$ Bodipy-C11 dye diluted in HBSS, followed by flow cytometry. Cells were gated for live and single cells.

Detection of GSH/GSSG. For detection of the GSH/GSSG ratio, cells were plated and treated for $24 \mathrm{~h}$ with non-starvation or starvation media. Then cells were washed with cold PBS and lysed with mammalian cell lysis buffer (Abcam, Cambridge, UK). GSH/GSSG ratio was measured with a commercially available kit (Abcam), according to the manufacturer's instructions. Samples were deproteinized before measurement.

Colony formation assay. Cells were plated at the indicated densities onto 6-well plates. $24 \mathrm{~h}$ after plating, cells were washed twice with PBS and incubated in starvation or non-starvation media for $72 \mathrm{~h}$, which was followed by a recovery period in normal growth medium. Antioxidants or pro-oxidants were added during the treatment period at the indicated concentrations. After the recovery period, cells were washed with PBS, fixed in methanol:acetic acid $(3: 1 \mathrm{v} / \mathrm{v})$ and stained using $0.4 \%$ crystal violet. The colony area was determined by using the Colony Area plugin [34] and ImageJ software (NIH).

Cell counting. 100,000 (H23) or 75,000 (A549) cells were plated and treated for $24 \mathrm{~h}$ with non-starvation or starvation medium and different concentrations of stabilized $\mathrm{H}_{2} \mathrm{O}_{2}$ (Roth, Karlsruhe, Germany). BSO (Sigma) was administered for $72 \mathrm{~h}$ according the duration of treatment in the colony formation experiments. Then cells were trypsinized and counted with the Casy-TT cell counter (Roche Innovatis, Bielefeld, Germany).

Proliferation. Cells were plated and treated $24 \mathrm{~h}$ with nonstarvation or starvation media supplemented with $10 \mathrm{mM}$ lactate. For detection of proliferative cells, the EdU Click-iT kit (Thermo Scientific) was utilized according to the manufacturer. In brief, EdU was added at a concentration of $10 \mu \mathrm{M}$ and incubated for $1.5 \mathrm{~h}$. Cells were collected, fixed, permeabilized and incubated with the freshly prepared Click iT reaction cocktail. EdU-positive cells were determined by FACS after gating for live and single cells.

Western blot. Protein was extracted with RIPA buffer (Thermo Scientific) and the concentration was determined by BCA assay (Merck, Vienna, Austria). Proteins were separated by sodium dodecyl sulfatepolyacrylamide gel electrophoresis using the Mini-PROTEAN ${ }^{\circledR}$ electrophoresis unit (BioRad, Hercules, CA) and transferred to a PVDF membrane (BioRad). The following antibodies were used: PCK2 (Abcam, ab187145; 1:2000), total OXPHOS antibody cocktail (Abcam, ab 110411, 1:1000), TOM20 (Cell Signaling Technology, \#42406, 1:1000), SLC7A11 (Abcam, ab175186, 1:1500) and $\beta$-actin (Santa Cruz, sc-47778 and Sigma, A5441). $\beta$-actin was used as a loading control.

Quantitative real-time PCR (RT-qPCR). Total RNA was extracted with the Qiagen RNeasy Mini kit (Qiagen, Hilden, Germany) and RNA concentration was determined by spectrophotometry. Total RNA was reverse transcribed with the $\mathrm{qScript} \mathrm{t}^{\mathrm{TM}} \mathrm{cDNA}$ Synthesis Kit (Quanta, Beverly, USA) according to the manufacturer's instructions. The QuantiFast SYBR PCR kit (Qiagen) was used to perform RT-qPCR on a LightCycler 480 (Roche, Vienna, Austria). Primers used were NFE2L2, 5'-TGCCAACTACTCCCAGGTTG-3' (forward), 5'-AAGTGACTGAAACGTAGCCGA-3' (reverse); GSR, 5'-CAGCGTCATTGTTGGTGCAG-3' (forward), 5'-CCTTGACCTGGGAGAACTTCAG-3' (reverse); SLC7A11, 5'TGACTGGAGTCCCTGCGTAT-3' (forward), 5'-TGTTCTGGTTATTT TCTCCGACATT-3' (reverse); TXNRD1, 5'-CGATCTGCCCGTTGTGTTTG3' (forward), 5'-TATTGGGCTGCCTCCTTAGC-3' (reverse); UCP2, 5'ACAAGACCATTGCACGAGAG-3' (forward), 5'-ATGAGGTTGGCTTTCAGGAG-3' (reverse); SOD2, 5'-CCTCACATCAACGCGCAGATCA-3' (forward), 5'-ACAACCTGAACGTCACCGAGGA-3' (reverse); GPX1, 5'CCAAGCTCATCACCTGGTCT-3' (forward), 5'-TCGATGTCAATGGTCT GGAA-3' (reverse); GPX4, 5'-TACCGGGGCTTCGTGTGCAT-3' (forward), 5'-TAGCCCGCGGCGAACTCTTT-3' (reverse); SRXN1, 5'CCTCGTGGACACGATCCG-3' (forward), 5'- 
CAGCCCCCAAAGGAGTAGAA-3' (reverse); GLRX, 5'-AACAGAGTGGGGAACTGCTG-3' (forward), 5'-TGAACATTTCCTATGAGATCTGTGG-3' (reverse); GSTP, 5'-GAGGGCTCACTCAAAGCCTC-3' (forward), 5'-GTCCTTCCCATAGAGCCCAAG-3' (reverse); GLS1, 5'GAGTTGCTGGGGGCATTCT-3' (forward), 5'-TACACAGAGAAACAAGATCGTGACA-3' (reverse); GLS2, 5'-GGCAGAGAGACGCCACA-3' (forward), 5'-CCTTTAGTGCAGTGGTGAACTT-3' (reverse); GLUD1, 5'CTCCAGACATGAGCACAGGTGA-3' (forward), 5'-CCAGTAGCAGAGATGCGTCCAT-3' (reverse); ACLY, 5'-TGCTCGATTATGCACTGGAAGT-3' (forward), 5'-ATGAACCCCATACTCCTTCCCAG-3' (reverse), PCK2, 5'-CATCCGAAAGCTCCCCAAGTA-3' (forward), 5'-TGGAAATCAGCTGGGGACATC-3' (reverse); АCTB, 5'-ATTGCCGACAGGATGCAGGAA-3' (forward), 5'-GCTGATCCACATCTGCTGGAA-3' (reverse). All primers were purchased from Eurofins Genomics. ACTB served as the reference gene since it showed stable expression over all samples. Melting curves were analyzed to verify the correct amplification of the expected RT-qPCR product. Cp values were determined with the 2 nd derivative method and the relative expression was calculated as $\Delta \mathrm{Cp}$.

Statistics. Data were analyzed with Microsoft Excel 2016 or SPSS, version 23.0 (Chicago, IL, USA). Group comparisons were made using two-sided, unpaired or paired Student's t-tests, one-group Student's ttests or one-way ANOVA with Dunnett post-hoc analysis as applicable, using data from at least three independent experiments. A p $<0.05$ was considered significant.

\section{Results}

\subsection{PCK2 suppresses TCA cycle activity and limits TCA cycle} intermediate abundance

The TCA cycle provides reducing equivalents to the respiratory chain. We assessed the abundance of TCA cycle intermediates and traced their interconversion in non-small cell lung cancer (NSCLC) cells by using uniformly ${ }^{13} \mathrm{C}$-labeled glutamine, the most important precursor for TCA cycle intermediates $[3,6]$. To mimic conditions of full nutrient availability, the medium was supplemented with $10 \mathrm{mM}$ glucose and $10 \%$ dialyzed fetal calf serum (dFCS). In contrast, a low concentration of glucose $(0.2 \mathrm{mM})$ in serum-free medium was used for experiments under starvation conditions. Serum was omitted in starvation media, since it contains lipids and other macromolecular nutrients. Under starvation conditions, H23 and A549 lung cancer cells, showed a moderate decrease in the TCA cycle intermediates fumarate or malate, and a decline in the total amount of citrate, compared to non-starvation conditions (Fig. 1A and Supplementary Fig. 1A). Glutamine provided carbons to TCA cycle intermediates under both conditions, leading to the full ${ }^{13} \mathrm{C}$ labeling of malate and fumarate (denoted as $\mathrm{M}+4$ ) (Fig. $1 \mathrm{~B}$ and C, Supplementary Figs. 1C and D). Accordingly, citrate $\mathrm{M}+4$ was generated from the condensation of fully labeled OAA with unlabeled acetyl-CoA. Upon treatment with starvation media, citrate $\mathrm{M}+6$ was formed from OAA $(M+4)$ and fully labeled acetyl-CoA $(M+2)$, which was very low under non-starvation conditions (Fig. 1B and C, Supplementary Figs. 1C and D). This indicates a higher rate of conversion of
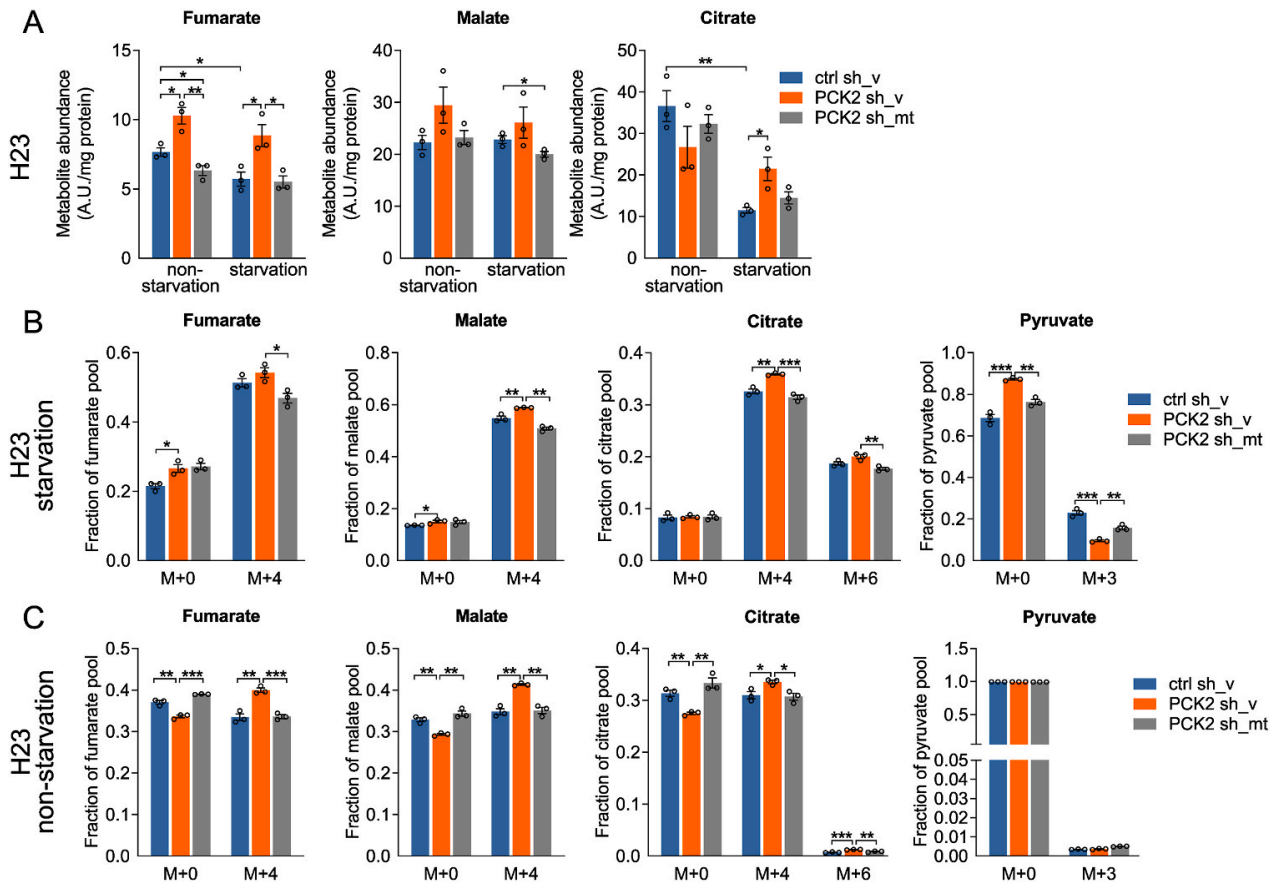

D

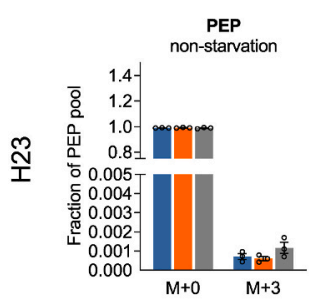

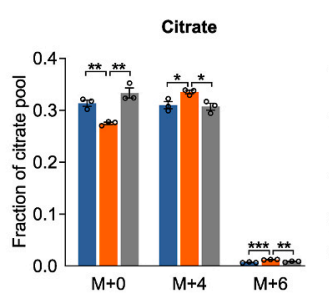

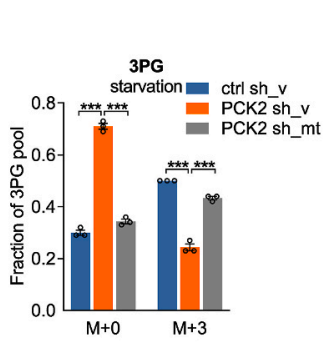

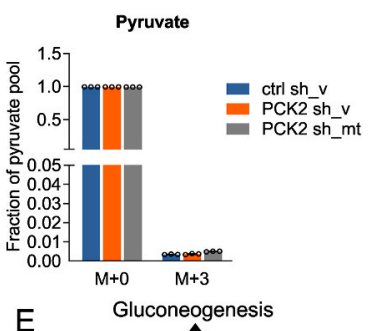

E

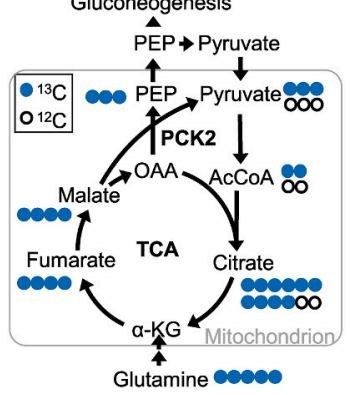

Fig. 1. PCK2 silencing enhances stable isotopic labeling and abundance of TCA cycle intermediates and reduces labeling of downstream gluconeogenesis products. A) Total abundances of TCA cycle intermediates of control (ctrl sh_v), PCK2 silenced (PCK2 sh_v) or PCK2 re-expressed (PCK2sh_mt) H23 cells after treatment with non-starvation medium or starvation medium B,C,D) Fractional enrichment of isotopologues of control, PCK2 silenced or PCK2 re-expressed $\mathrm{H} 23$ cells treated with starvation medium B,D) or non-starvation medium C,D) containing ${ }^{13} \mathrm{C}_{5}$-glutamine. E) Metabolic pathway for the conversion of ${ }^{13} \mathrm{C}_{5}$-glutamine into TCA cycle and gluconeogenesis intermediates. Results are shown as mean \pm SEM from $\mathrm{n}=3$ independent experiments. Group comparisons were made using two-sided, unpaired Student's t-tests. *p $<0.05,{ }^{* *} \mathrm{p}<0.01, * * * \mathrm{p}<0.001$. PEP, phosphoenolpyruvate; 3 PG, 3-phosphoglycerate; A.U., arbitrary units. 
TCA cycle metabolites via pyruvate to acetyl-CoA under treatment with starvation which has been already described to occur in absence of glucose [35], mediated either via PEPCK or malic enzyme (ME). Accordingly, a significant proportion of pyruvate, the precursor of acetyl-CoA, was fully ${ }^{13} \mathrm{C}$ labeled $(\mathrm{M}+3)$ in starvation, but not in non-starvation medium (Fig. 1B and C, Supplementary Figs. 1C and D). A scheme of possible labeling patterns of TCA cycle intermediates, after the addition of ${ }^{13} \mathrm{C}_{5}$-glutamine, is depicted in Fig. $1 \mathrm{E}$.

In order to address the role of PCK2 in tuning the TCA cycle under starvation conditions, PCK2 was silenced by stable expression of PCK2 shRNA (PCK2 sh). PCK2 knock-down was rescued by the expression of a point mutated, PCK2 shRNA resistant allele (PCK2 sh_mt; Supplementary Fig. 2B). Alternatively, PCK2 was silenced with two different siRNA pools (Supplementary Fig. 2A). Re-expression of PCK2 in A549 cells was performed by simultaneous overexpression of wild-type PCK2 in PCK2 silenced cells (Supplementary Fig. 2C). When total levels of TCA cycle intermediates were assessed, we found that PCK2 silencing clearly increased the levels of fumarate, malate and citrate under starvation conditions in both cell lines (Fig. 1A and Supplementary Fig. 1A). The effect was blunted by the re-expression of shRNA resistant PCK2 in H23 cells (Fig. 1A) and by the parallel overexpression of wild-type PCK2 in PCK2 silenced A549 cells (Supplementary Fig. 1B). In H23 cells, M+4 labeling of citrate and malate was slightly increased by PCK2 silencing (Fig. 1B). The effects were partly also observed at the level of fumarate. Likewise, silencing of PCK2 in A549 cells by two different siRNA pools led to an increased abundance of $\mathrm{M}+4$ isotopologues of malate and fumarate and enhanced $\mathrm{M}+6$ labeling of citrate under starvation conditions (Supplementary Fig. 1C). In H23 cells, the fraction of pyruvate $\mathrm{M}+3$ was decreased by PCK2 silencing (Fig. 1B), while it was slightly enhanced in A549 cells (Supplementary Fig. 1C). These results indicate that PCK2 contributes to OAA decarboxylation in H23 cells, but not in A549 cells. The latter may utilize a different route of TCA cycle carbon to pyruvate conversion, e.g. via ME. Together, these data demonstrate that PCK2 removes OAA from the TCA cycle under starvation conditions, leading to a reduced abundance and interconversion of TCA cycle intermediates.

Importantly, the initial steps in glutamine carbon utilization, the conversion of glutamine to glutamate and the further transamination to $\alpha$-ketoglutarate ( $\alpha$-KG), remained unaffected by PCK2 silencing when treated with starvation media (Supplementary Figs. 3A and B). Under non-starvation conditions, the fraction of fully labeled glutamate and $\alpha-K G(M+5), M+4$ fumarate, malate and citrate and also the absolute abundance of $\alpha$-KG and fumarate were increased by PCK2 silencing in H23 (Fig. 1A and C, Supplementary Figs. 3A and C) but not in A549 cells (Supplementary Figs. 3B and D). This may be related to a slightly, but not significantly enhanced expression of the initial enzyme in glutamine utilization, glutaminase (GLS1) and a slight decrease in the expression of the cataplerotic enzyme ATP citrate lyase (ACLY) in H23 cells upon PCK2 silencing under non-starvation conditions (Supplementary Fig. 3E).

PCK2 contributed to the gluconeogenesis pathway under starvation conditions, as shown by the high rate of conversion of ${ }^{13} \mathrm{C}_{5}$-glutamine via OAA to PEP. Between 40 and $50 \%$ of PEP showed a full labeling by ${ }^{13} \mathrm{C}$ and a similar enrichment was found at the level of the downstream gluconeogenesis intermediate 3-phosphoglycerate (3 PG) (Fig. 1D and Supplementary Fig. 1E). Only a small fraction of PEP $(0.1-1 \%)$ was labeled under treatment with non-starvation media, showing that PCK2 activity in the direction of gluconeogenesis was low under these conditions. This was accompanied by a reduced expression of PCK2 (Supplementary Fig. 2A), similar to our previous findings [16,19]. Importantly, PCK2 silencing decreased the fraction of ${ }^{13} \mathrm{C}$-labeled PEP and 3 PG (Fig. 1D and Supplementary Fig. 1E). In A549 cells, starvation treatment increased the abundance of PEP whereas it was reduced by PCK2 silencing (Supplementary Fig. 1E).

PCK2 decreases mitochondrial respiration under starvation conditions. The TCA cycle produces reducing equivalents, which are oxidized in the electron transport chain (ETC) to generate ATP [36]. When we measured oxygen consumption rates (OCR), we found an increase in mitochondrial respiration under starvation compared to non-starvation conditions (Fig. 2A-G). Starvation-induced basal respiration was further clearly enhanced upon silencing of PCK2 (Fig. 2B-G). In both cell lines, PCK2 silencing increased both, basal and maximal OCR, but not ATP-linked respiration under treatment with starvation media, suggesting that the additional oxygen consumed under PCK2-silenced conditions is not utilized for ATP biosynthesis (Fig. 2D-G). No significant effect of PCK2 silencing was found under non-starvation conditions (Fig. 2D-G). During OCR measurements either pyruvate or lactate were added as a respiratory fuel. Of note, the cells respired also in the absence of pyruvate or lactate (starvation-lac, Fig. 2B), with a similar enhancement by PCK2 silencing compared to cells under starvation media supplemented with lactate. Treatment with etomoxir (Eto), an inhibitor of fatty acid oxidation, decreased the level of OCR under starvation conditions, indicating that in the absence of glucose and serum, cells partially utilize (endogenous) fatty acids to fuel respiration (Fig. 2C). Also if fatty oxidation was blocked, PCK2 silenced cells showed elevated oxygen consumption rates (Fig. 2C). These data indicate that mitochondrial respiration is decreased by PCK2.

Mitochondrial morphology can vary under different nutritional conditions. Mitochondria tend to be elongated in case of inappropriate nutrient supply as this protects them from autophagosomal degradation and induces increased oxidative phosphorylation (OXPHOS) [37,38]. When mitochondrial morphology was visualized by Mitotracker green, the number of individual mitochondria did not differ between starvation and non-starvation conditions (Supplementary Fig. 4A). However, PCK2 silencing decreased the number of individual mitochondria under starvation conditions (Supplementary Fig. 4A). Mitochondrial mass was not significantly affected, neither by treatment with starvation media nor by PCK2 silencing (Supplementary Fig. 4B). Taken together these findings suggest that PCK2 silencing leads to mitochondrial elongation. In order to clarify, whether an upregulated expression of complex members of the respiratory chain causes enhanced mitochondrial respiration under PCK2 silencing, we assessed the protein abundance of key electron transport chain subunits. The expression of NDUFB8 (complex I), SDHB (complex II), UQCRC2 (complex III), COX II (complex IV) and ATP5A (F1 subunit of complex V) and the mitochondrial protein TOM20 remained unchanged (Supplementary Figs. $4 \mathrm{C}$ and D). Thus, enhancement of respiration by PCK2 silencing occurs rather due to modulation of the TCA cycle activity than due to altered expression of OXPHOS members.

PCK2 improves the redox balance in starved lung cancer cells. The respiratory chain is the major source of potentially harmful ROS which need to be continuously scavenged by different antioxidant enzymes at the expense of NADPH and GSH $[8,13,14]$. We found that different antioxidant genes NFE2L2, GSR and the cystine transporter subunit SLC7A11 were up-regulated in NSCLC cells after 24 h of treatment with starvation media (Fig. 3A). With PCK2 si1, PCK2 silencing led to a slight but significant suppression of starvation-induced SLC7A11 expression in both cell lines, however this was not consistent over all silencing approaches (Fig. 3A). When we analyzed SLC7A11 protein levels we did not find any modulation by PCK2 silencing (Fig. 3B).

Interrogating, if the increased activity of the mitochondrial respiration, observed under starvation conditions and triggered by PCK2 silencing, leads to an enhanced formation of ROS, we measured mitochondrial superoxide and cellular ROS. Treatment with starvation media resulted in a slight increase in mitochondrial superoxide, which was not significantly altered by PCK2 silencing (Fig. 4A). Likewise, DCFDA oxidation, a marker of increased ROS levels, showed only a small, insignificant increase under starvation conditions and PCK2 silencing (Fig. 4B). However, PCK2 silencing under starvation conditions significantly decreased the ratio of reduced to oxidized GSH (GSH/GSSG), as shown by two different methods (Fig. 4C and Supplementary Fig. 5A). Moreover, the NADPH/NADP ${ }^{+}$ratio was decreased upon PCK2 silencing 
A

Basal oxygen consumption

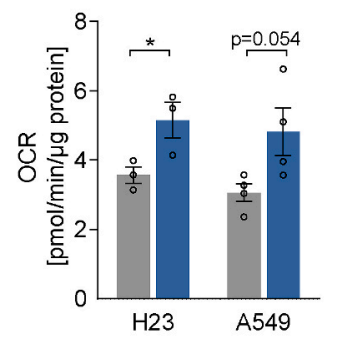

D
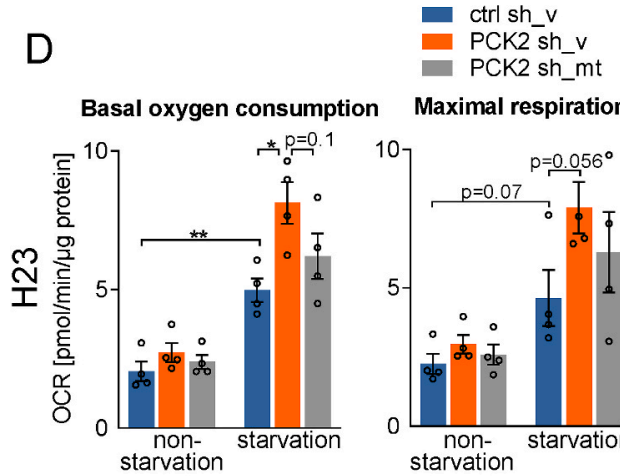

$\mathrm{F}$

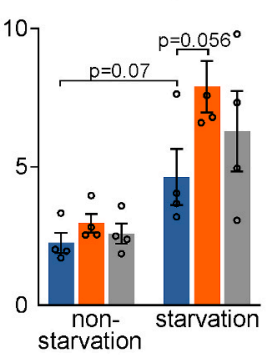

$$
\begin{aligned}
& \text { ctrl si } \\
& \text { PCK2 si1 }
\end{aligned}
$$

Basal oxygen consumption Maximal respiration

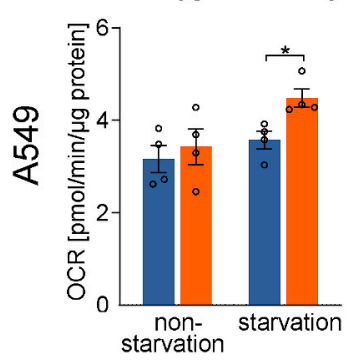

B

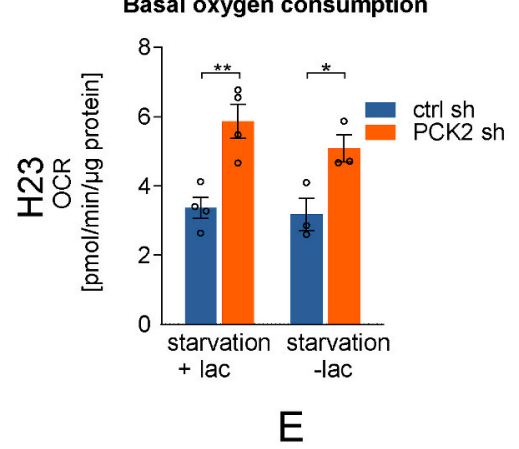

C

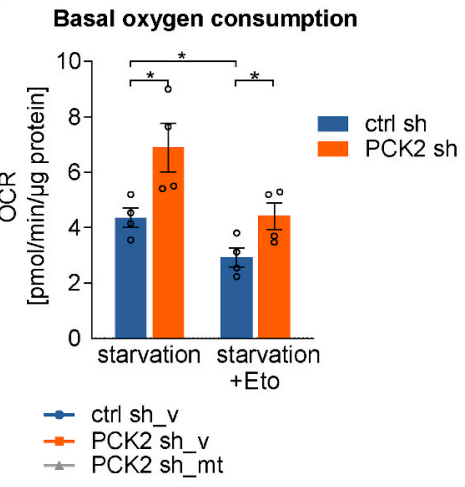

ATP production

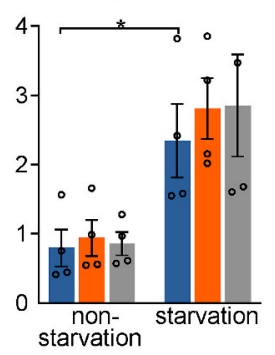

starvation

G
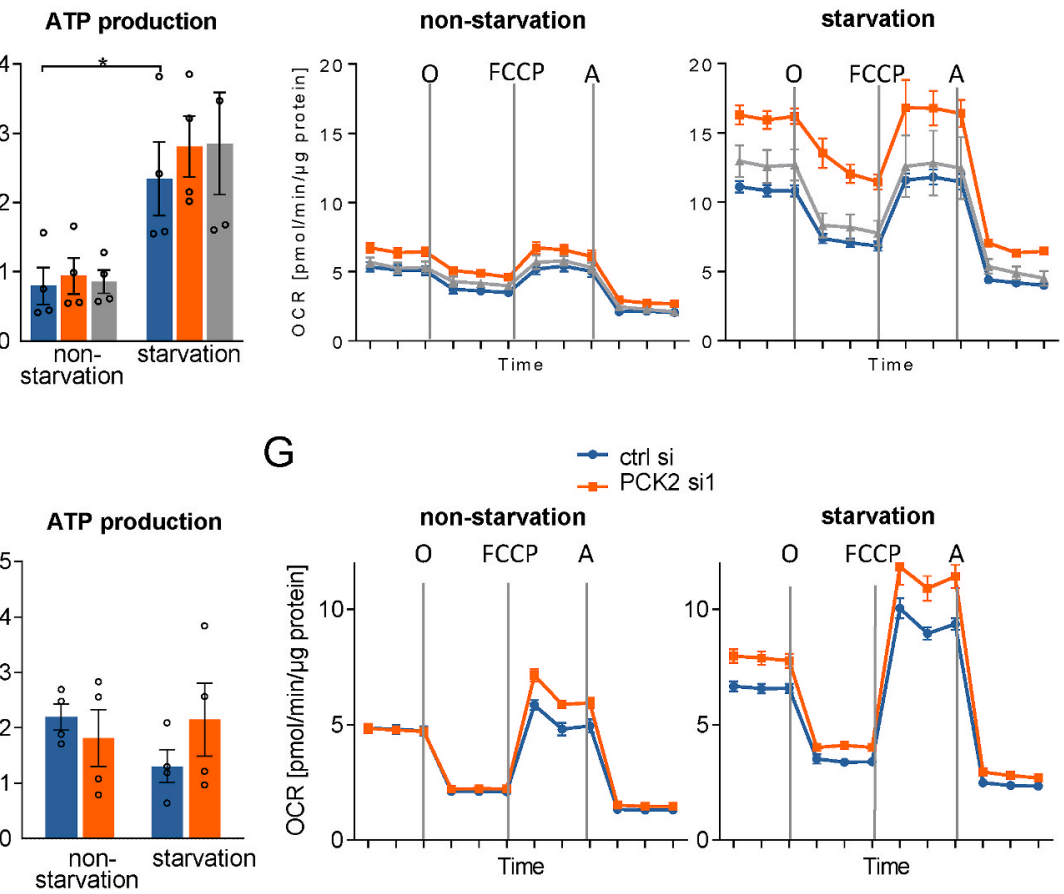

Fig. 2. PCK2 silencing enhances cellular respiration. A) Basal oxygen consumption rate (OCR) in H23 or A549 cells treated with non-starvation medium or starvation medium. B) OCR of control (ctrl sh) or PCK2 silenced (PCK2 sh) H23 cells, cultured in starvation medium in the presence or absence of lactate. C) OCR of control (ctrl sh) or PCK2 silenced (PCK2 sh) H23 cells, cultured in starvation medium in the presence or absence of etomoxir (Eto). D,F) Basal and maximal OCR and ATPproduction-linked OCR in control (ctrl sh_v), PCK2 silenced (PCK2 sh_v) or PCK2 re-expressed (PCK2sh_mt) H23 cells or in control (ctrl si) or PCK2 silenced (PCK2 si1) A549 cells. Cells were treated with starvation or non-starvation medium. E,G) Representative OCR recordings over time at basal levels and after the addition of oligomycin (O), FCCP or antimycin (A), showing mean values \pm SEM of five or six technical replicates. A-D,F) Results are shown as mean \pm SEM from four independent experiments, each consisting of four to six technical replicates. Comparison between groups was performed using two-sided, unpaired Student's t-tests. $* \mathrm{p}<0.05, * * \mathrm{p}<0.01$.

under treatment with starvation media (Fig. 4D). Lipid peroxidation is a self-perpetuating detrimental oxidative process that may lead to a specific, iron dependent, form of cell death, ferroptosis [14]. It is efficiently controlled by the lipid specific enzyme glutathione peroxidase 4 (GPX4) which reduces lipid peroxidation by utilizing reduced GSH [39]. If the GPX4 antioxidant system was blocked by the GPX4 inhibitor RSL3, PCK2 silencing caused significantly enhanced lipid peroxidation levels, indicating a higher burden of ROS and/or an insufficiency of alternative antioxidant defense mechanisms (Fig. 4E). An enhanced expression of antioxidant enzymes under starvation conditions and a decreased GSH/GSSG ratio suggest that ROS formed by the ETC under PCK2 silencing may be scavenged by antioxidant defense mechanisms at the expense of a diminished glutathione redox capacity. In $\mathrm{H} 23$ cells, but not A549 cells, GSH depletion induced by PCK2 silencing was also observed under non-starvation conditions (Fig. 4C). Oxygen consumption was only insignificantly increased under these conditions (Fig. 2D and E), despite the enhanced abundance of TCA cycle intermediates in this cell line (Fig. 1A). Potentially, ROS formation from enhanced TCA cycle intermediates, independent of a forward oxidation in the respiratory chain, might play a role, e.g. succinate-fueled reverse-electron flow [40].

In order to clarify whether increased TCA activity and subsequently enhanced respiration were responsible for the GSH/GSSG imbalance, we utilized dimethyl-L-malate (DMM), a membrane permeable analogue of the TCA cycle intermediate malate. Fueling the TCA cycle with $5 \mathrm{mM}$ DMM mimicked the effects of PCK2 silencing on respiration and depleted GSH in $\mathrm{H} 23$ cells, while not increasing superoxide (Fig. 4F). Partly, these effects were also observed in A549 cells (Supplementary Figs. 6A-C). These data show that PCK2 activity diminishes oxidative stress and contributes to maintaining the GSH/GSSG redox balance by reducing respiration via suppression of the TCA cycle.

GSH levels are regenerated by the reduction of GSSG, however, GSH levels are also maintained by de novo synthesis from glycine, cysteine and glutamate [41]. Interestingly, PCK2 silencing was associated with a 
A
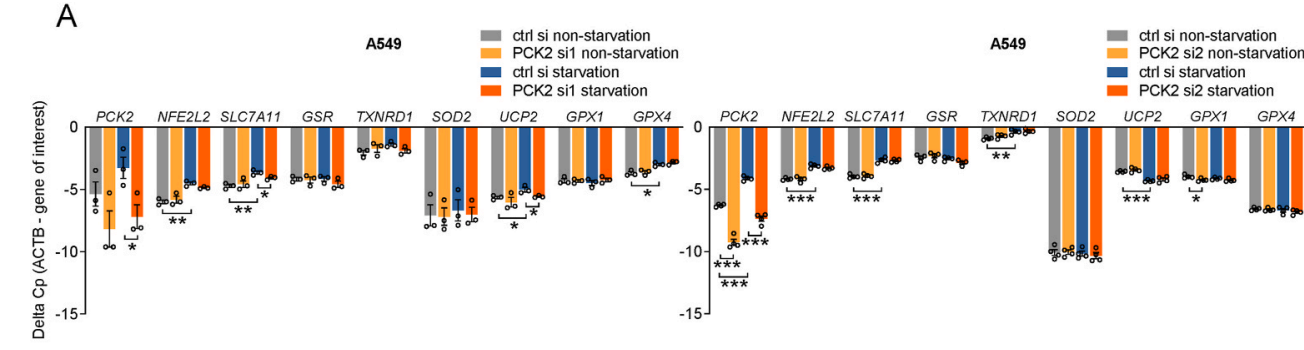

XNRD1 SOD2 UCP2 GPX1 $\quad$ GPX4
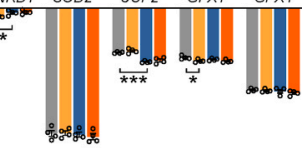

H23

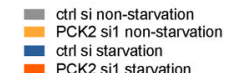
PCK2 si1 ation

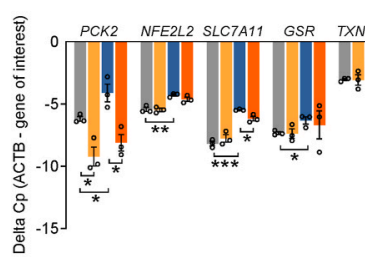
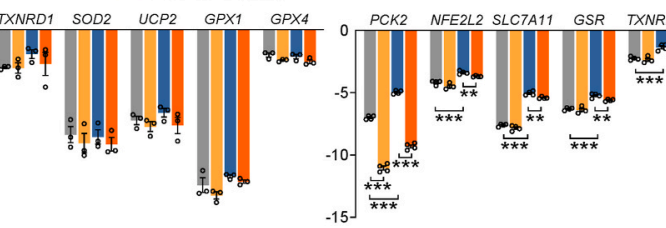

H23

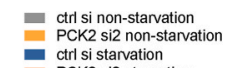
- ctrl si starvation

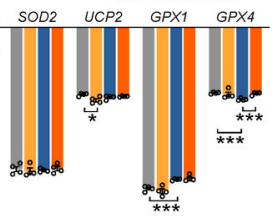

B
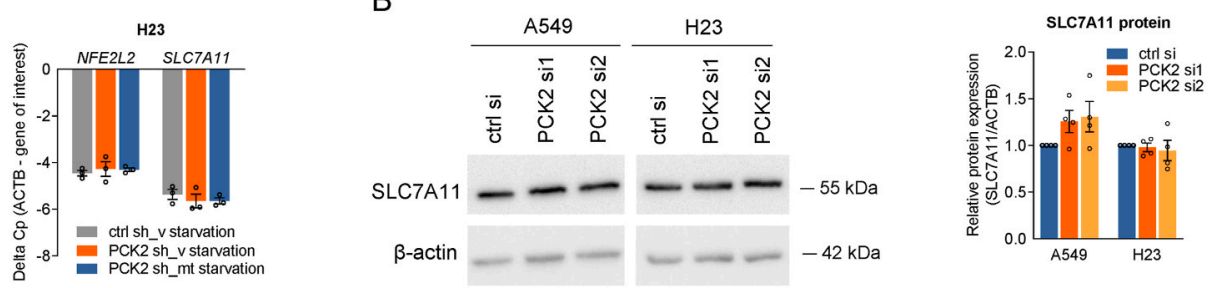

A

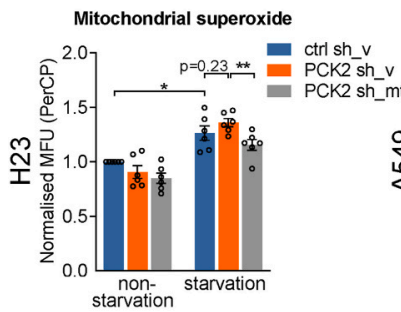

c

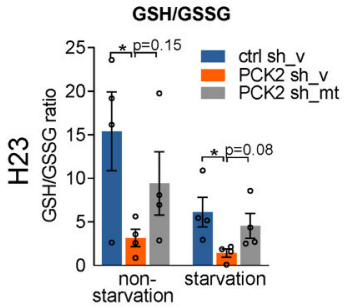

F Basal oxygen consumption

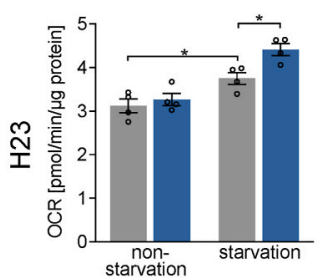

B
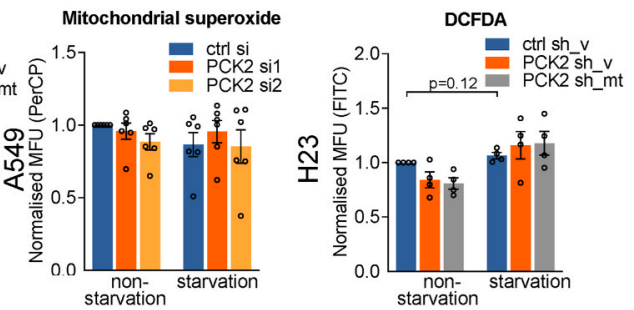

D
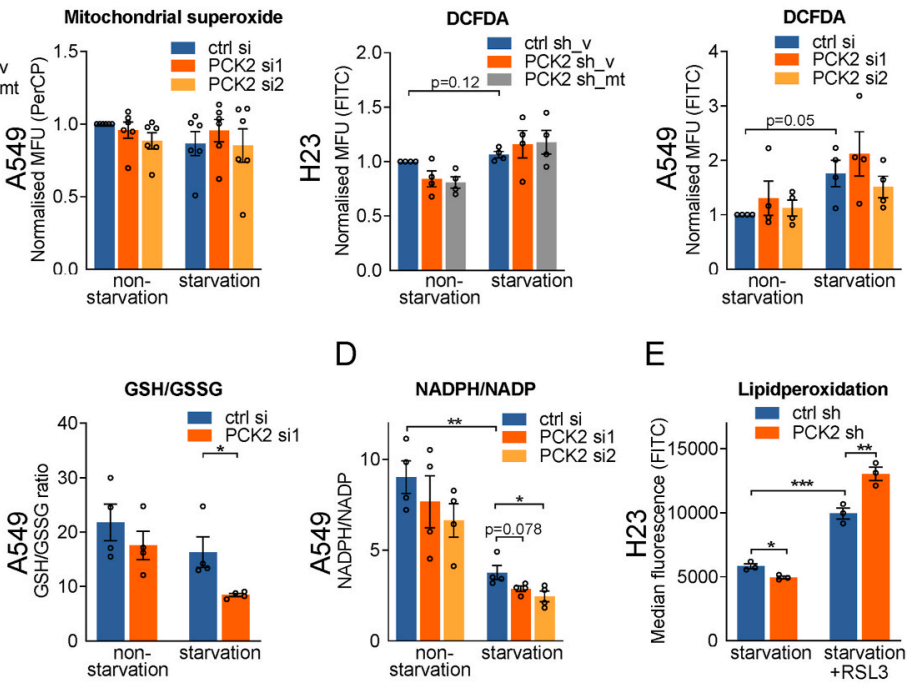

E
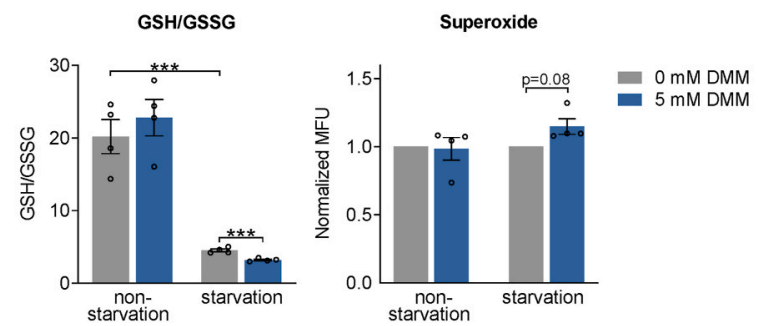

Fig. 3. Starvation increases the expression of antioxidant genes, partly modulated by PCK2 silencing. A) Expression of PCK2 and redox genes (normalized to $\beta$-actin, ACTB) in control (ctrl si) or PCK2 silenced (PCK2 si1, PCK2 si2) H23 and A549 cells, treated with non-starvation or starvation media and of control (ctrl sh_v), PCK2 silenced (PCK2 sh_v) or PCK2 re-expressed (PCK2sh_mt) H23 cells treated with starvation media. B) Control or PCK2 silenced A549 or H23 cells were treated with starvation media and SLC7A11 expression was analyzed by Western blot. Representative blots and densitometric analysis are shown. $\beta$-actin served as a loading control. Results are mean \pm SEM from three (PCK2 si1, PCK2 sh) or four (PCK2 si2, B)) independent experiments. Group comparisons were made using twosided, unpaired Student's t-tests or one group Student's t-test as applicable. *p < $0.05, * * \mathrm{p}<0.01, * * * \mathrm{p}<0.001$.
Fig. 4. PCK2 silencing substantially decreases the ratio of reduced to oxidized glutathione, dimethyl-L-malate addition mimics PCK2 silencing. A) Mitochondrial superoxide in control (ctrl sh_v), PCK2 silenced (PCK2 sh_v) or PCK2 re-expressed (PCK2 sh_mt) H23 cells or in control (ctrl si) or PCK2 silenced (PCK2 si1/2) A549 cells, treated with non-starvation or starvation medium; $\mathrm{n}=6$ independent experiments. B) Oxidation of CM- $\mathrm{H}_{2}$ DCFDA in $\mathrm{H} 23$ and A549 cells treated as described in A); $\mathrm{n}$ $=4$ independent experiments. C) Reduced (GSH) to oxidized (GSSG) glutathione ratio in $\mathrm{H} 23$ and A549 cells treated as described in A); $\mathrm{n}=4$ independent experiments. D) Ratio of NADPH to NADP measured by LC-MS/MS in A549 cells treated as described in A); $n=$ 4 independent experiments. E) Lipid peroxidation in control (ctrl sh) or PCK2 silenced (PCK2 sh) H23 cells treated with starvation medium with or without glutathione peroxidase 4 inhibitor RSL3; $n=3$ independent experiments. F) H23 cells were treated with non-starvation or starvation media containing $5 \mathrm{mM}$ dimethyl-L-malate (DMM). Basal oxygen consumption, GSH/GSSG ratio and mitochondrial superoxide production were measured; $\mathrm{n}=4$ independent experiments. Results are shown as mean \pm SEM. Comparison between groups was performed using two-sided, unpaired Student's t-tests or one group Student's t-test as applicable. ${ }^{*} \mathrm{p}<0.05,{ }^{* *} \mathrm{p}<0.01, * * * \mathrm{p}<0.001$. 
slightly reduced rate of GSH de novo synthesis, as shown by the lower fractional abundance of GSH M+5, in starved H23 but not A549 cells (Supplementary Fig. 5B). The M+5 labeled GSH found under starvation conditions likely reflects labeled glutamate (carrying 5 carbons), which is directly formed from ${ }^{13} \mathrm{C}$-glutamine. We could not detect a transfer of ${ }^{13} \mathrm{C}$ from glutamine to serine/glycine in $\mathrm{H} 23$ cells and a low level of transfer in A549 cells, under our experimental conditions (data not shown). Accordingly, we did not detect higher isotopologues in the GSH pool under these experimental conditions. These data indicate that PCK2 slightly promotes GSH de novo biosynthesis in $\mathrm{H} 23$ cells independent of glycine biosynthesis.

PCK2 promotes colony formation under starvation conditions and reduces the sensitivity towards $\mathrm{H}_{2} \mathrm{O}_{2}$. Next, we investigated colony formation by lung cancer cells under starvation or non-starvation conditions. After an initial starvation or non-starvation treatment, we allowed cells to recover and form colonies in full growth medium. PCK2 silencing significantly diminished colony formation of loosely seeded cells under starvation (Fig. 5A). In H23 cells the colony forming ability was also reduced under non-starvation treatment. No effect of PCK2 silencing on proliferation or cell numbers was found in densely seeded cells under starvation conditions (Fig. 5B and C). We examined whether PCK2 expression protects starved cancer cells from damage induced by exogenous $\mathrm{H}_{2} \mathrm{O}_{2}$. In both cell lines, PCK2 silencing significantly enhanced the toxic effects of $\mathrm{H}_{2} \mathrm{O}_{2}$ under treatment with starvation (Fig. $5 \mathrm{C}$ ). In order to investigate whether the reduced colony formation by PCK2 silencing is caused by the redox imbalance, we added different antioxidants simultaneously with starvation or non-starvation treatment. Trolox, a derivative of vitamin E, exogenous GSH, as well as the antioxidant and GSH precursor $N$-acetyl cysteine (NAC) all rescued the effect of reduced colony forming ability in PCK2 silenced cells (Fig. 6A and B). Importantly, enhancing TCA cycle intermediates by using the malate analogue DMM mimicked the impact of PCK2 silencing on colony formation in $\mathrm{H} 23$ but not in A549 cells (Fig. 6C and Supplementary Fig. 6D). The generally less pronounced effects of DMM supplementation in A549 cells might be attributed to a higher efflux/decarboxylation of TCA intermediates through ME in that cell line, which generates mitochondrial NADPH. As we found a striking contrast between the effect of PCK2 silencing on cell proliferation and cell numbers in densely plated cells and PCK2 silencing under colony formation conditions we wanted to clarify, if loosely plated, colony forming cancer cells are more sensitive towards oxidation. We used buthionine sulfoximine (BSO), an inhibitor of GSH biosynthesis and inducer of oxidative stress. In fact, treatment with different concentrations of BSO highly reduced the cellular colony forming ability upon non-starvation and starvation conditions, whereas it affected cell numbers in densely seeded cells to a much lower extent (Fig. 7A and B).

Addition of the protein glutathionylation agent diamide mimics PCK2 silencing. GSSG-induced modifications, independent of direct cellular damage by ROS, might contribute to the suppressive effect of PCK2 silencing on colony formation. High GSSG and low GSH levels have been found to promote S-glutathionylation of proteins [42]. To address the question whether protein S-glutathionylation may be responsible for PCK2 silencing induced reduction of colony formation, we added diamide, an S-glutathionylating agent, to starvation or non-starvation media. Diamide concentration-dependently caused a decrease in the colony forming ability under treatment with starvation, but not non-starvation media (Fig. 7C), indicating that starvation conditions render colony forming cancer cells vulnerable towards glutathionylation of proteins. Additionally, enzymes responsible for protein de-glutathionylation, such as sulfiredoxin (SRXN1) and glutaredoxin-1 (GLRX), but not glutathione S-transferase P (GSTP), which selectively glutathionylates proteins, were up-regulated upon starvation conditions in both cell lines (Fig. 7D).

In summary, these results demonstrate that the cataplerotic action of PCK2 directly reduces TCA cycle intermediate abundance and interconversion, diminishes mitochondrial respiration and depletes cellular GSH. The rescue by antioxidants and the phenocopy by a protein glutathionylating agent suggest that PCK2-mediated maintenance of the GSH/GSSG balance is important during colony formation under nutrient starvation. A model for the protective role of PCK2 in mediating cataplerosis, limiting respiration and maintaining the glutathione redox balance in cancer cells is proposed in Fig. 8.

\section{Discussion}

In many tumor types, the utilization of certain steps of gluconeogenesis is beneficial in starved cancer cells, since this pathway yields building blocks for biomass production [27]. The TCA cycle is a metabolic hub, feeding into biosynthetic pathways for the generation of amino acids, nucleic acids or fatty acids, but the cycle also produces reducing equivalents, critical for electron transport chain and ATP production $[3,11,36]$. Here, we identify the importance of the gluconeogenesis enzyme PCK2 as a regulator of the TCA cycle, limiting mitochondrial respiration and enhancing the antioxidant defense in

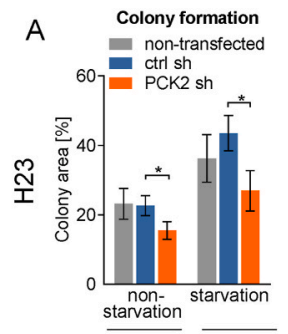

Plated/well $\overline{750 \text { cells }} \overline{5000 \text { cells }}$
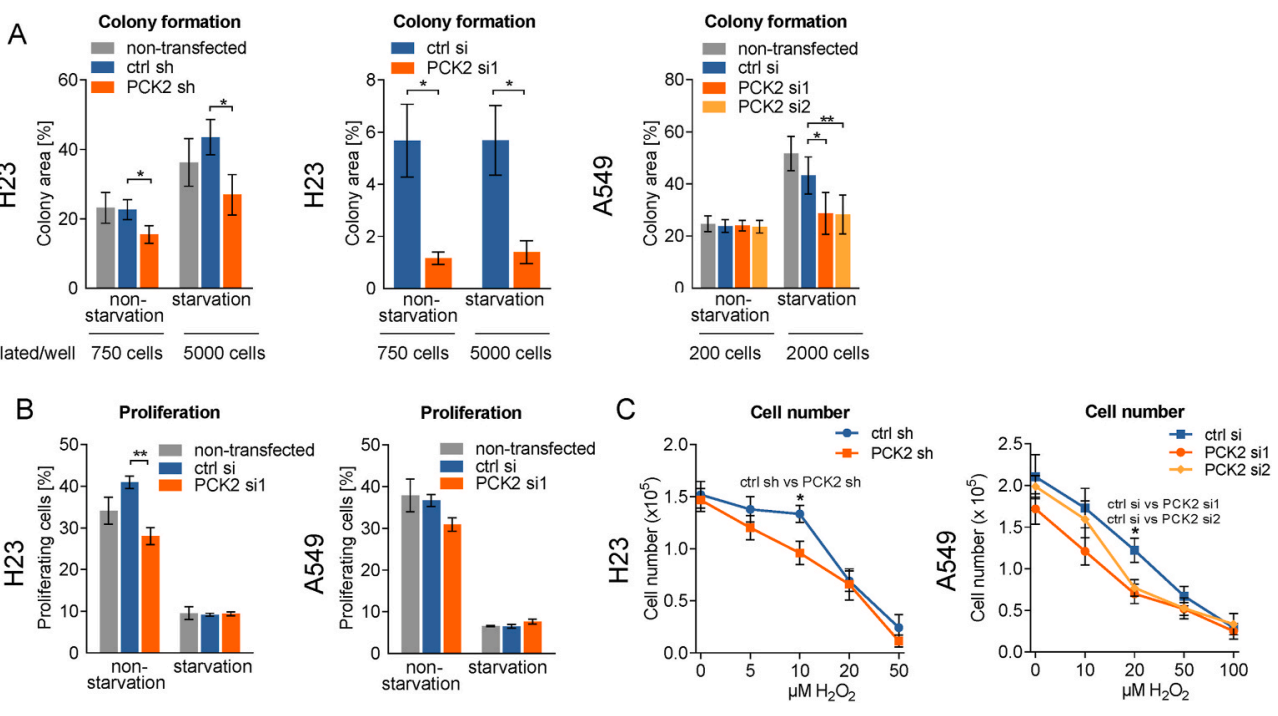

Fig. 5. PCK2 silencing reduces colony forming ability and sensitizes cancer cells $\mathrm{H}_{2} \mathrm{O}_{2}$. A) $\mathrm{H} 23$ or A549 cells were plated for colony formation and treated with nonstarvation (nstv) or starvation (stv) media followed by a recovery in normal growth media prior to the assessment of colony area. $\mathrm{n}=8$ (H23 shRNA), $\mathrm{n}=4$ (H23 siRNA), $\mathrm{n}=$ 12 (A549) individual experiments, each performed in technical triplicates. Data are mean \pm SEM. Group comparisons were made using two-sided, paired Student's ttests B) EdU incorporation into densely plated control or PCK2 silenced cells, treated with non-starvation or starvation media, $\mathrm{n}$ $=3$ individual experiments. $\mathrm{C}$ ) Cell counts of densely plated control or PCK2 silenced cells, treated with starvation media containing different concentrations of $\mathrm{H}_{2} \mathrm{O}_{2} ; n$ $=4$ individual experiments, each performed in technical duplicates. B,C) Data are mean \pm SEM. Group comparisons were made using two-sided, unpaired Student's t-tests. ${ }^{*} \mathrm{p}<$ $0.05, * * \mathrm{p}<0.01$. 
A

$\mathrm{H} 23$
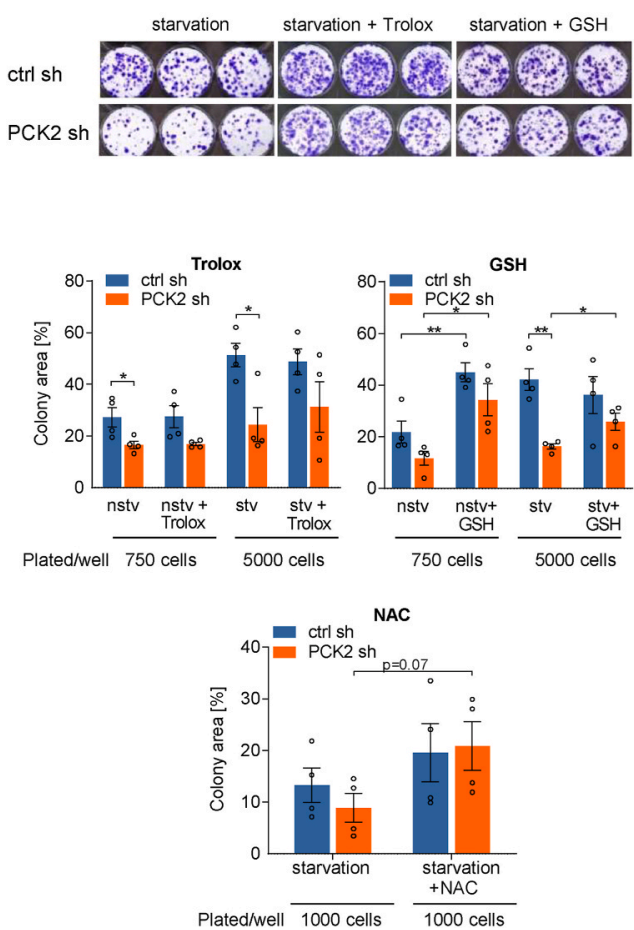

B

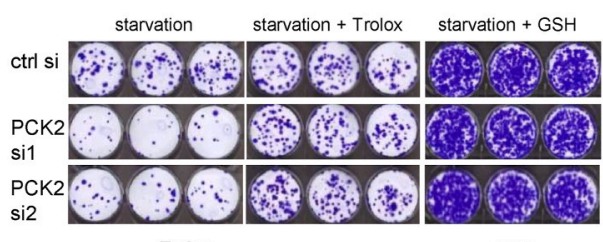

Trolox
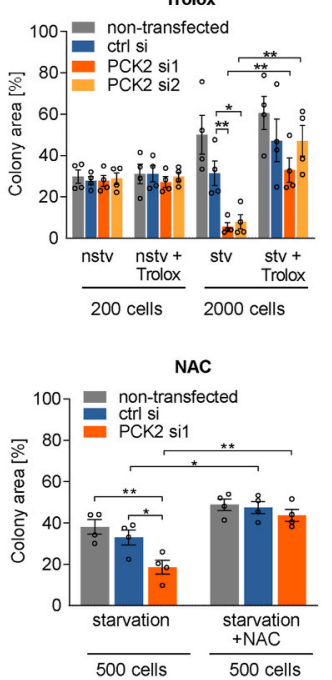

A549

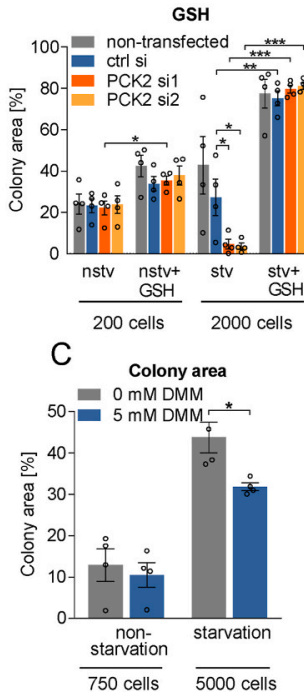

Fig. 6. PCK2 silencing reduces colony forming capability of H23 and A549 cells, rescued by antioxidants, phenocopy by dimethylmalate. A) H23 cells expressing control shRNA or a PCK2 shRNA were plated for colony formation and treated with nonstarvation (nstv) or starvation (stv) media followed by a recovery in normal growth media. The antioxidants Trolox $(100 \mu \mathrm{M})$, glutathione (GSH, $2 \mathrm{mM}$ ), or $N$-acetyl cysteine (NAC, $10 \mathrm{mM}$ ) were added simultaneously with nstv or stv treatment. B) A549 cells were non-transfected or transfected with non-silencing (ctrl si) or PCK2 silencing siRNA (PCK2 si1/2) and treated as above with or without Trolox $(100 \mu \mathrm{M}), \mathrm{GSH}$ (2 mM), or NAC (5 mM). C) H23 cells were plated and treated with nstv/stv media as described above. During the treatment period, $5 \mathrm{mM}$ dimethyl-L-malate (DMM) was added. Data are shown as mean \pm SEM from four independent experiments, consisting out of three technical replicates. Group comparisons were made using two-sided, unpaired Student's t-tests. *p $<0.05$, **p $<0.01,{ }^{* * *} \mathrm{p}<0.001$.
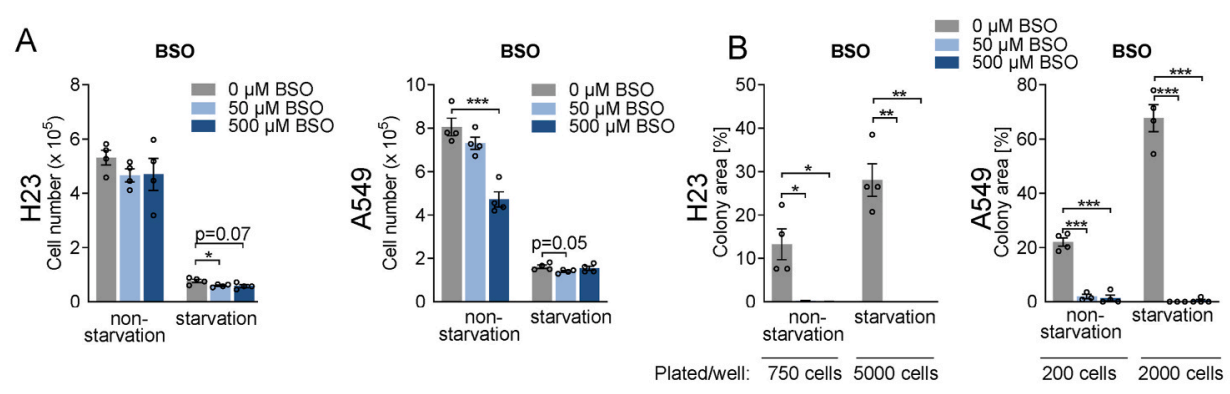

C
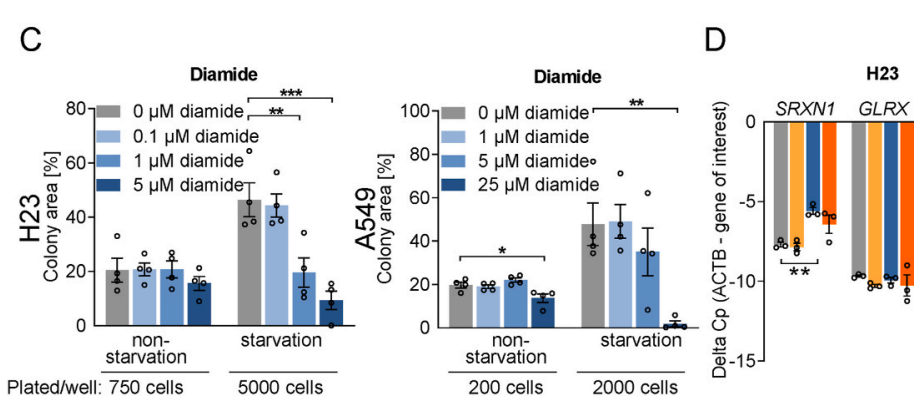

Fig. 7. Inhibition of colony formation by glutathione synthesis inhibitor buthionine sulfoximine (BSO) and diamide, a glutathionylating agent. A) Cell counts of densely plated A549 or H23 cells treated for $72 \mathrm{~h}$ with different concentrations of BSO in nonstarvation or starvation media. B) Colony forming capability of A549 or H23 cells treated for $72 \mathrm{~h}$ with different concentrations of BSO in non-starvation or starvation media, followed by recovery in normal growth media. C) H23 and A549 cells were plated for colony formation assay and treated with non-starvation or starvation media in the presence or absence of diamide. D) Expression of enzymes modifying glutathionylation, sulfiredoxin (SRXN1), glutaredoxin-1 (GLRX) and glutathione Stransferase $\mathrm{P}$ (GSTP), normalized to $\beta$-actin $(A C T B)$. Data are shown as mean \pm SEM from three D) or four independent experiments, consisting out of three $\mathrm{B}, \mathrm{C}$ ) or two A) technical replicates. A,B,D) Group comparisons were made using two-sided Student's ttests and C) using one-way ANOVA and Dunnett post hoc analysis. * $\mathrm{p}<0.05$, ${ }^{* *} \mathrm{p}<$ $0.01, * * * \mathrm{p}<0.001$. nutrient-starved lung cancer cells.

We show that PCK2 silencing causes increased abundance of TCA cycle intermediates, especially in low glucose, serum-free media. Accordingly, we found augmented mitochondrial respiration under starvation conditions, which got even enhanced by PCK2 silencing. Electron leakage from the ETC causes the formation of ROS, which are scavenged by antioxidant enzymes at the expense of GSH [13]. Treatment with starvation media induced the antioxidant genes NFE2L2, SLC7A11 and GSR. The enhancement of NFE2L2 and SLC7A11 by starvation has been previously described [43]. Many antioxidant systems, including glutathione peroxidases, utilize GSH as a co-factor [13]. In fact, the GSH/GSSG ratio and consequently the NADPH/NADP ${ }^{+}$ratio were decreased by PCK2 silencing under treatment with starvation media. ROS levels remained unchanged by PCK2 silencing under these experimental conditions, showing that ROS were continuously scavenged by the antioxidant systems, at the expense of reduced glutathione. However, when lipid peroxidation was initiated by addition of a GPX4 inhibitor, PCK2 silencing led to an increased level of oxidized phospholipids. In summary, these data demonstrate that PCK2 plays an important role in maintaining the cellular antioxidant defense in lung 


\section{PCK2 expressing cancer cell}

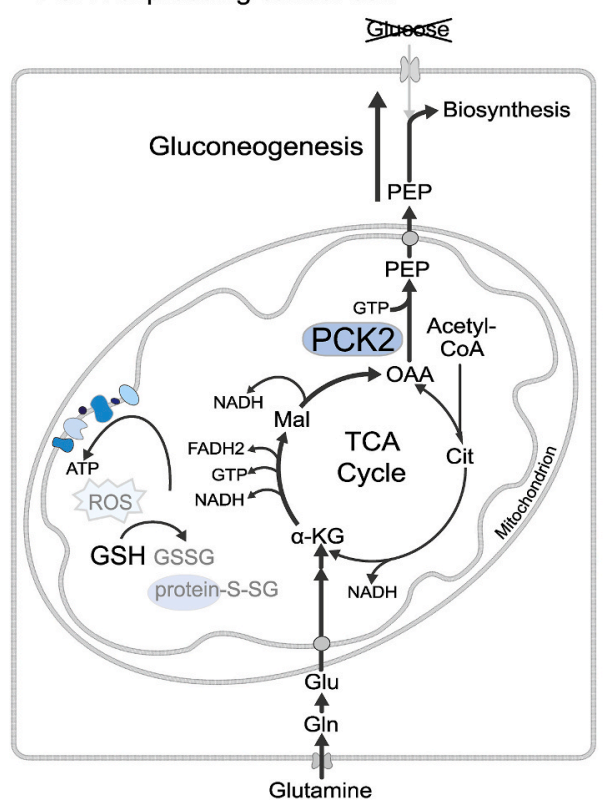

PCK2 silenced cancer cell

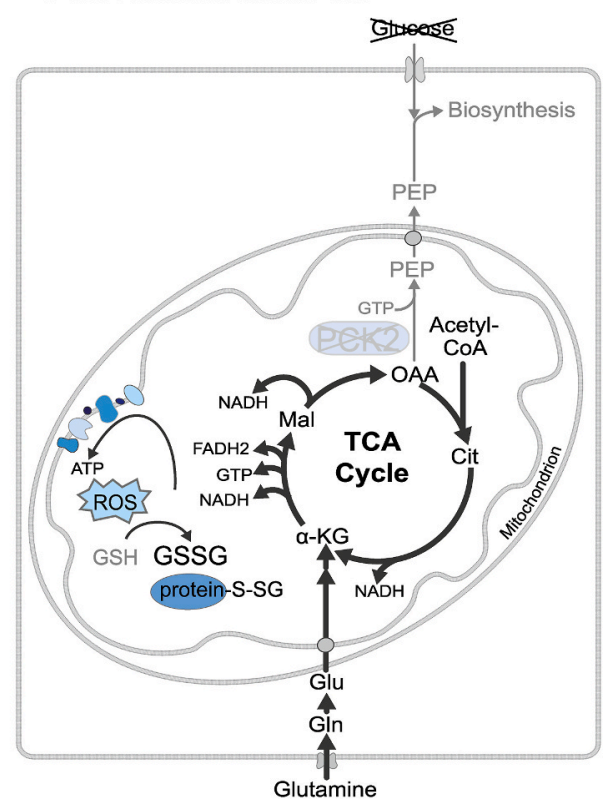

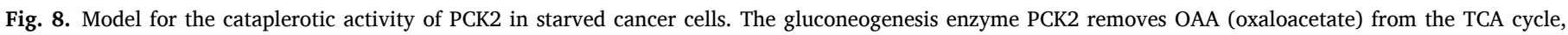
especially under starvation conditions. Thereby, PCK2 suppresses mitochondrial respiration and maintains a reduced glutathione redox potential.

cancer upon treatment with starvation media.

We noticed a slight but significant suppression of SLC7A11 mRNA by PCK2 si1 upon starvation, however, no regulation of SLC7A11 on the protein level occurred. In addition to modulation on a transcriptional level, SLC7A11 abundance has been previously shown to be also regulated by post-translational mechanisms or by interaction with its binding partner SLC3A2 [44], which might explain the discrepancy of mRNA and protein levels. Taken together, these data suggest that regulation of SLC7A11 does not play a major role in the observed antioxidative effect of PCK2. In addition to the prevention of the accumulation of free ROS, a decreased GSH/GSSG ratio provokes modifications of signaling proteins by S-glutathionylation e.g. protein kinase $\mathrm{C}$ or nuclear factor (NF)-кB, thereby altering their function [42]. Of note, we found a highly increased expression of sulfiredoxin or GLRX, crucial, GSH-dependent deglutathionylating enzymes, under starvation conditions, possibly related to an enhanced rate of protein-glutathionylation.

The role of PCK2 in tumor cells, pro- or antitumorigenic, has been previously linked to its effects on the TCA cycle, although the role of PCK2 under glucose and serum starvation has not been addressed in these studies. Under high glucose conditions, PCK2 silencing has been found to increase TCA cycle intermediate abundance in tumor initiating cells of melanoma and prostate cancer, as well as in hypoxic breast cancer cells $[20,45,46]$. However, the effect of PCK2 on the incorporation of ${ }^{13} \mathrm{C}_{5}$-glutamine into the TCA cycle was not analyzed. While TCA cycle suppression by PCK2 and the decrease in citrate were associated with enhanced proliferation in prostate cancer cells due to diminished protein acetylation and superoxide levels [20], the same effect of PCK2 on the TCA cycle was linked to growth inhibition in melanoma cells [45]. In breast cancer cells, inhibition of the TCA cycle and suppression of ROS formation by PCK2 overexpression appeared to cause a growth arrest [46]. In contrast, PCK2 inhibition rather than activation caused a decrease in TCA cycle flux in cervix carcinoma stress under abundant glucose [28]. In both, low and high glucose conditions PCK2 inhibition led to an increase in ROS formation in that model, which was attributed to an increase in proline degradation by proline dehydrogenase, an enzyme that generates ROS as a byproduct [28]. In glucose but not serum starved A549 cells, PCK2 silencing did not significantly alter glutamine derived anaplerosis of the TCA cycle [18], however, in contrast to our study, media containing $0 \mathrm{mM}$ glucose and $10 \% \mathrm{dFCS}$ were utilized and abundances of isotopologues were normalized to high glucose conditions. PCK1 overexpression in liver cancer cells was shown to reduce TCA metabolite levels due to cataplerosis by PEPCK in high and low glucose containing media, thereby enhancing ROS formation and reducing cell viability due to an energy crisis [47]. In contrast, PCK1 silencing reduced the abundance of ${ }^{13} \mathrm{C}_{5}$ glutamine or ${ }^{13} \mathrm{C}_{3}$ lactate derived TCA cycle intermediates in colon cancer cells $[48,49]$. However, to the best of our knowledge, the impact of PCK1 or PCK2 on mitochondrial respiration in nutrient deprived cancer cells remained unknown. Our study mechanistically links the cataplerotic activity of PCK2 to the repression of mitochondrial respiration and consequently to an enhanced availability of reduced glutathione in starved lung cancer cells. As a limitation, the relevance of these PCK2-mediated metabolic effects for its tumor growth promoting role in vivo [18,19] is still undetermined and should be addressed in future studies.

Given the variable effects of PCK1/2 on TCA cycle in different studies and systems, the question arises, whether different rates of the TCA cycle in different tissues of origin may determine the overall effect of PCK1/2 on TCA cycle intermediate abundance and TCA cycle flux. In noncancerous liver and intestine, PCK1 knockout mice displayed an increase in TCA cycle intermediates connected to decreased cataplerosis [50-53]. In contrast to our findings obtained in lung cancer cells, the cataplerotic effect of PCK1 rather enhanced than repressed TCA cycle flux and respiration in liver from mice fed a high fat diet [53]. This was attributed to a reduced feedback inhibition by NADH [53]. Thus, the effect of PCK1 and PCK2 on the TCA cycle and on mitochondrial respiration might be context-dependent and related to the energy/NADH status of the mitochondria.

The multifaceted role of the TCA cycle as a metabolic hub that controls anabolic pathways and ROS production gains further complexity by the fact that it may promote the production of NADPH either directly by $\mathrm{NADP}^{+}$utilizing isoforms of isocitrate dehydrogenase (e.g. IDH2), or indirectly by feeding intermediates into the ME pathway $[3,6]$. In our study, we found that promoting the TCA cycle in lung cancer cells, either by PCK2 silencing or by exogenous addition of DMM, enhanced respiration and decreased the GSH/GSSG redox potential. The data are in line with a previous report on a dose dependent enhancement of mitochondrial respiration and ROS formation by DMM in lung cancer cells [54]. The up-regulation of antioxidant defense mechanisms, e.g. 
the induction of the NRF2 target gene SLC7A11 by starvation conditions found in our study and in previous reports [43], indicates that low nutrient supply is associated with a higher burden of ROS in cancer cells. Glucose deprivation as well as complete nutrient starvation mediated by Hank's balanced salt solution (HBSS) treatment were shown to trigger ROS formation [43,55-57]. When lipid oxidation was measured in tumor-bearing mice fed with a ketogenic, low-carbohydrate diet, an increase in lipid peroxidation was found upon radiation therapy and supporting the efficacy of the treatment [58]. The reason for enhanced burden of ROS under glucose deprivation conditions is not completely understood, however increased respiration or reduced regeneration/biosynthesis of antioxidant molecules, such as NADPH or GSH, might play a role.

Our study suggests an important role in TCA cycle cataplerosis by PCK2 to balance respiration and ROS formation under starvation conditions. Interestingly, PCK2 silencing did not enhance ROS formation and proliferation in cells growing in high confluency, but dramatically reduced colony formation under starvation conditions. We found that loosely seeded, colony forming cells are more susceptible towards oxidative stress compared to densely seeded cells. It has been shown previously that certain cell types have enhanced ROS levels upon loose seeding densities [59]. In line with an enhanced dependency of colony forming cells on antioxidant systems, GSH and its precursor NAC did not only rescue decreased colony forming ability by PCK2 silencing but increased colony formation upon treatment with starvation media also in control cells. Importantly, both, the TCA cycle intermediate DMM and the protein glutathionylating agent diamide phenocopied the effects of PCK2 silencing. Together, these data demonstrate that the redox balancing effect of PCK2 is beneficial for survival and colony formation of starved lung cancer cells. The phenotypic changes in cancer cells underlying the inhibition of colony formation induced by PCK2 silencing and GSH-loss remain unclear and should be investigated in future studies. Besides ROS-induced cell death, a diminished proliferation due to alterations in cell signaling might play a role. It has been previously shown that PCK2 mediates the generation of glycerol phosphate for phospholipid backbone biosynthesis in glucose starved lung cancer cells and that exogenous phosphatidylethanolamine (PE) phospholipids partially rescue colony formation inhibited by PCK2 knockdown [19]. Of note, PE, especially when containing polyunsaturated fatty acids, is highly susceptible to peroxidation [60]. Thus, PE backbone synthesis and turnover, facilitated by PCK2, might act together with the TCA suppressing effects of PCK2 described here to reduce ROS induced alterations under starvation conditions.

\section{Conclusions}

In summary, we show that PCK2 diminishes the TCA cycle and thus reduces starvation-induced mitochondrial respiration in starved lung cancer cells, maintaining the glutathione redox balance. This balance, however, is crucial for colony formation capabilities especially under starvation conditions and protects cells from the attack by exogenous ROS. Thus, PCK2 inhibition represents a potential new therapeutic approach to prevent metabolic adaptation and distort the redox balance in lung cancer cells.

\section{Funding}

The study was supported by the Austrian Science Fund (P 33508 to K. L.). G.B. received funding from a DOC Fellowship of the Austrian Academy of Sciences (25282). The study was further supported by DKMCD W1226 (to W.F.G.), by the Austrian Science Fund Erwin Schroedinger Abroad Fellowship (J4205-B27 to C.T.M.), and by MEFO Graz (to K.L. and W.F.G.). S.-M.F. acknowledges funding from the European Research Council under the ERC Consolidator Grant Agreement n. 771486-MetaRegulation, FWO (Research Foundation - Flanders) Research Projects, KU Leuven - Methusalem Co-Funding and Fonds
Baillet Latour.

\section{Declaration of competing interest}

The authors declare that they have no conflict of interest.

\section{Acknowledgments}

We thank A. Bertsch and D. Broekaert (VIB-KU Leuven) for excellent technical support and we are grateful for the valuable advice by G. Höfler and T. Madl (Medical University of Graz).

\section{Appendix A. Supplementary data}

Supplementary data to this article can be found online at https://doi. org/10.1016/j.freeradbiomed.2021.09.007.

\section{Author contributions}

G.B., C.T.M., W.F.G., S.-M.F., H.O. and K.L. designed research; G.B., M.P., C.T.M., T.H., A.H. and K.L. performed research; G.B., M.P., C.T.M., W.F.G., S.-M.F., and. K.L., analyzed data; G.B. and K.L. wrote the paper.

\section{References}

[1] M.G. Vander Heiden, L.C. Cantley, C.B. Thompson, Understanding the warburg effect: the metabolic requirements of cell proliferation, Science 324 (2009) 1029-1033, https://doi.org/10.1126/science.1160809.

[2] A. Schulze, A.L. Harris, How cancer metabolism is tuned for proliferation and vulnerable to disruption, Nature 491 (2012) 364-373, https://doi.org/10.1038/ nature11706.

[3] R.J. DeBerardinis, T. Cheng, Q's next: the diverse functions of glutamine in metabolism, cell biology and cancer, Oncogene 29 (2010) 313-324, https://doi. org/10.1038/onc.2009.358.

[4] P. Vaupel, Tumor microenvironmental physiology and its implications for radiation oncology, Semin. Radiat. Oncol. 14 (2004) 198-206, https://doi.org/10.1016/j. semradonc.2004.04.008.

[5] M.R. Sullivan, L.V. Danai, C.A. Lewis, S.H. Chan, D.Y. Gui, T. Kunchok, E. A. Dennstedt, M.G. Vander Heiden, A. Muir, Quantification of microenvironmental metabolites in murine cancers reveals determinants of tumor nutrient availability, Elife 8 (2019), e44235, https://doi.org/10.7554/eLife.44235.

[6] R.J. DeBerardinis, N.S. Chandel, Fundamentals of cancer metabolism, Sci. Adv. 2 (2016), e1600200, https://doi.org/10.1126/sciadv.1600200.

[7] R.A. Cairns, I.S. Harris, T.W. Mak, Regulation of cancer cell metabolism, Nat. Rev. Cancer. 11 (2011) 85-95, https://doi.org/10.1038/nrc2981.

[8] N.S. Chandel, Navigating Metabolism, Cold Spring Harbor Laboratory Press, Cold Spring Harbor, NY, 2015.

[9] N.N. Pavlova, C.B. Thompson, The emerging hallmarks of cancer metabolism, Cell. Metab. 23 (2016) 27-47, https://doi.org/10.1016/j.cmet.2015.12.006.

[10] H.A. Krebs, Rate control of the tricarboxylic acid cycle, Adv. Enzyme Regul. 8 (1970) 335-353, https://doi.org/10.1016/0065-2571(70)90028-2.

[11] O.E. Owen, S.C. Kalhan, R.W. Hanson, The key role of anaplerosis and cataplerosis for citric acid cycle function, J. Biol. Chem. 277 (2002) 30409-30412, https://doi. org/10.1074/jbc.R200006200.

[12] K. Birsoy, R. Possemato, F.K. Lorbeer, E.C. Bayraktar, P. Thiru, B. Yucel, T. Wang, W.W. Chen, C.B. Clish, D.M. Sabatini, Metabolic determinants of cancer cell sensitivity to glucose limitation and biguanides, Nature 508 (2014) 108-112, https://doi.org/10.1038/nature13110.

[13] C.R. Reczek, N.S. Chandel, ROS-dependent signal transduction, Curr. Opin. Cell Biol. 33 (2015) 8-13, https://doi.org/10.1016/j.ceb.2014.09.010.

[14] I.S. Harris, G.M. DeNicola, The complex interplay between antioxidants and ROS in cancer, Trends Cell Biol. 30 (2020) 440-451, https://doi.org/10.1016/j. tcb.2020.03.002.

[15] H. Sasaki, H. Sato, K. Kuriyama-Matsumura, K. Sato, K. Maebara, H. Wang, M. Tamba, K. Itoh, M. Yamamoto, S. Bannai, Electrophile response elementmediated induction of the cystine/glutamate exchange transporter gene expression, J. Biol. Chem. 277 (2002) 44765-44771, pii: M208704200.

[16] K. Leithner, A. Hrzenjak, M. Trotzmuller, T. Moustafa, H.C. Kofeler, C. Wohlkoenig, E. Stacher, J. Lindenmann, A.L. Harris, A. Olschewski, et al., PCK2 activation mediates an adaptive response to glucose depletion in lung cancer, Oncogene 34 (2015) 1044-1050, https://doi.org/10.1038/onc.2014.47.

[17] A. Mendez-Lucas, P. Hyrossova, L. Novellasdemunt, F. Vinals, J.C. Perales, Mitochondrial phosphoenolpyruvate carboxykinase (PEPCK-M) is a pro-survival, endoplasmic reticulum (ER) stress response gene involved in tumor cell adaptation to nutrient availability, J. Biol. Chem. 289 (2014) 22090-22102, pii: M114.566927.

[18] E.E. Vincent, A. Sergushichev, T. Griss, M.C. Gingras, B. Samborska, T. Ntimbane, P.P. Coelho, J. Blagih, T.C. Raissi, L. Choiniere, et al., Mitochondrial 
phosphoenolpyruvate carboxykinase regulates metabolic adaptation and enables glucose-independent tumor growth, Mol. Cell 60 (2015) 195-207, https://doi.org/ 10.1016/j.molcel.2015.08.013.

[19] K. Leithner, A. Triebl, M. Trotzmuller, B. Hinteregger, P. Leko, B.I. Wieser, G. Grasmann, A.L. Bertsch, T. Zullig, E. Stacher, et al., The glycerol backbone of phospholipids derives from noncarbohydrate precursors in starved lung cancer cells, Proc. Natl. Acad. Sci. U. S. A. 115 (2018) 6225-6230, https://doi.org/ 10.1073/pnas.1719871115.

[20] J. Zhao, J. Li, T.W.M. Fan, S.X. Hou, Glycolytic reprogramming through PCK2 regulates tumor initiation of prostate cancer cells, Oncotarget 8 (2017) 83602-83618, https://doi.org/10.18632/oncotarget.18787.

[21] S.Y. Chun, C. Johnson, J.G. Washburn, M.R. Cruz-Correa, D.T. Dang, L.H. Dang, Oncogenic KRAS modulates mitochondrial metabolism in human colon cancer cells by inducing HIF-1alpha and HIF-2alpha target genes, Mol. Cancer. 9 (2010) 293-4598, https://doi.org/10.1186/1476-4598-9-293, 9-293.

[22] P.Y. Chu, S.S. Jiang, Y.S. Shan, W.C. Hung, M.H. Chen, H.Y. Lin, Y.L. Chen, H. J. Tsai, L.T. Chen, Mitochondrial phosphoenolpyruvate carboxykinase (PEPCK-M) regulates the cell metabolism of pancreatic neuroendocrine tumors (pNET) and desensitizes pNET to mTOR inhibitors, Oncotarget 8 (2017) 103613-103625, https:// doi.org/10.18632/oncotarget.21665.

[23] E.I. Chen, J. Hewel, J.S. Krueger, C. Tiraby, M.R. Weber, A. Kralli, K. Becker, J. R. Yates 3rd, B. Felding-Habermann, Adaptation of energy metabolism in breast cancer brain metastases, Cancer Res 67 (2007) 1472-1486, pii: 67/4/1472.

[24] N.V. Chaika, F. Yu, V. Purohit, K. Mehla, A.J. Lazenby, D. DiMaio, J.M. Anderson, J.J. Yeh, K.R. Johnson, M.A. Hollingsworth, et al., Differential expression of metabolic genes in tumor and stromal components of primary and metastatic loci in pancreatic adenocarcinoma, PloS One 7 (2012), e32996, https://doi.org/ 10.1371/journal.pone.0032996.

[25] R. Stark, R.G. Kibbey, The mitochondrial isoform of phosphoenolpyruvate carboxykinase (PEPCK-M) and glucose homeostasis: has it been overlooked? Biochim. Biophys. Acta 1840 (2014) 1313-1330, https://doi.org/10.1016/j. bbagen.2013.10.033.

[26] E. Smolle, P. Leko, E. Stacher-Priehse, L. Brcic, A. El-Heliebi, L. Hofmann, F. Quehenberger, A. Hrzenjak, H.H. Popper, H. Olschewski, et al., Distribution and prognostic significance of gluconeogenesis and glycolysis in lung cancer, Mol. Oncol. 11 (2020) 2853-2867, https://doi.org/10.1002/1878-0261.12780.

[27] G. Grasmann, E. Smolle, H. Olschewski, K. Leithner, Gluconeogenesis in cancer cells - repurposing of a starvation-induced metabolic pathway? Biochim. Biophys. Acta Rev. Cancer. 1872 (2019) 24-36, https://doi.org/10.1016/j. bbcan.2019.05.006.

[28] P. Hyroššová, M. Aragó, J. Moreno-Felici, X. Fu, A. Mendez-Lucas, P.M. GarcíaRovés, S. Burgess, A. Figueras, F. Viñals, J.C. Perales, PEPCK-M recoups tumor cell anabolic potential in a PKC- $\zeta$-dependent manner, Cancer. Metab. 9 (2021) 1-3, https://doi.org/10.1186/s40170-020-00236-3, 020-00236.

[29] R. Keshet, J.S. Lee, L. Adler, M. Iraqi, Y. Ariav, L.Q.J. Lim, S. Lerner, S. Rabinovich, R. Oren, R. Katzir, et al., Targeting purine synthesis in ASS1-expressing tumors enhances the response to immune checkpoint inhibitors, Nature Cancer 1 (2020) 894-908, https://doi.org/10.1038/s43018-020-0106-7.

[30] D. Lorendeau, G. Rinaldi, R. Boon, P. Spincemaille, K. Metzger, C. Jager, S. Christen, X. Dong, S. Kuenen, K. Voordeckers, et al., Dual loss of succinate dehydrogenase (SDH) and complex I activity is necessary to recapitulate the metabolic phenotype of SDH mutant tumors, Metab. Eng. 43 (2017) 187-197, https://doi.org/10.1016/j.ymben.2016.11.005.

[31] P. Millard, B. Delepine, M. Guionnet, M. Heuillet, F. Bellvert, F. Letisse, IsoCor: Isotope correction for high-resolution MS labeling experiments, Bioinformatics 35 (2019) 4484-4487, https://doi.org/10.1093/bioinformatics/btz209.

[32] K.R. Kampen, L. Fancello, T. Girardi, G. Rinaldi, M. Planque, S.O. Sulima, F. Loayza-Puch, B. Verbelen, S. Vereecke, J. Verbeeck, et al., Translatome analysis reveals altered serine and glycine metabolism in T-cell acute lymphoblastic leukemia cells, Nat. Commun. 10 (2019) 2542, https://doi.org/10.1038/s41467. 019-10508-2.

[33] A.J. Valente, L.A. Maddalena, E.L. Robb, F. Moradi, J.A. Stuart, A simple ImageJ macro tool for analyzing mitochondrial network morphology in mammalian cell culture, Acta Histochem. 119 (2017) 315-326, pii: S0065-1281(16)30365-8.

[34] C. Guzman, M. Bagga, A. Kaur, J. Westermarck, D. Abankwa, ColonyArea: an ImageJ plugin to automatically quantify colony formation in clonogenic assays, PloS One 9 (2014), e92444, https://doi.org/10.1371/journal.pone.0092444.

[35] C. Yang, B. Ko, C.T. Hensley, L. Jiang, A.T. Wasti, J. Kim, J. Sudderth, M. A. Calvaruso, L. Lumata, M. Mitsche, et al., Glutamine oxidation maintains the TCA cycle and cell survival during impaired mitochondrial pyruvate transport, Mol. Cell 56 (2014) 414-424, https://doi.org/10.1016/j.molcel.2014.09.025.

[36] I. Martínez-Reyes, N.S. Chandel, Mitochondrial TCA cycle metabolites control physiology and disease, Nat. Commun. 11 (2020) 102, https://doi.org/10.1038/ s41467-019-13668-3.

[37] A.S. Rambold, B. Kostelecky, N. Elia, J. Lippincott-Schwartz, Tubular network formation protects mitochondria from autophagosomal degradation during nutrient starvation, Proc. Natl. Acad. Sci. U. S. A. 108 (2011) 10190-10195, https://doi.org/10.1073/pnas.1107402108.

[38] P. Mishra, D.C. Chan, Metabolic regulation of mitochondrial dynamics, J. Cell Biol. 212 (2016) 379-387, https://doi.org/10.1083/jcb.201511036.

[39] W.S. Yang, R. SriRamaratnam, M.E. Welsch, K. Shimada, R. Skouta, V. S. Viswanathan, J.H. Cheah, P.A. Clemons, A.F. Shamji, C.B. Clish, et al., Regulation of ferroptotic cancer cell death by GPX4, Cell 156 (2014) 317-331, https://doi.org/10.1016/j.cell.2013.12.010.

[40] E.T. Chouchani, V.R. Pell, E. Gaude, D. Aksentijevic, S.Y. Sundier, E.L. Robb, A. Logan, S.M. Nadtochiy, E.N.J. Ord, A.C. Smith, et al., Ischaemic accumulation of succinate controls reperfusion injury through mitochondrial ROS, Nature 515 (2014) 431-435, https://doi.org/10.1038/nature13909.

[41] A. Bansal, M.C. Simon, Glutathione metabolism in cancer progression and treatment resistance, J. Cell Biol. 217 (2018) 2291-2298, https://doi.org/ $10.1083 /$ jcb. 201804161.

[42] J.J. Mieyal, M.M. Gallogly, S. Qanungo, E.A. Sabens, M.D. Shelton, Molecular mechanisms and clinical implications of reversible protein S-glutathionylation, Antioxid. Redox Signal. 10 (2008) 1941-1988, https://doi.org/10.1089/ ars.2008.2089.

[43] P. Koppula, Y. Zhang, J. Shi, W. Li, B. Gan, The glutamate/cystine antiporter SLC7A11/xCT enhances cancer cell dependency on glucose by exporting glutamate, J. Biol. Chem. 292 (2017) 14240-14249, https://doi.org/10.1074/jbc. M117.798405.

[44] P. Koppula, Y. Zhang, L. Zhuang, B. Gan, Amino acid transporter SLC7A11/xCT at the crossroads of regulating redox homeostasis and nutrient dependency of cancer, Cancer. Commun. (Lond) 38 (2018) 12-18, https://doi.org/10.1186/s40880-0180288-x, 0288-x.

[45] S. Luo, Y. Li, R. Ma, J. Liu, P. Xu, H. Zhang, K. Tang, J. Ma, N. Liu, Y. Zhang, et al., Downregulation of PCK2 remodels tricarboxylic acid cycle in tumor-repopulating cells of melanoma, Oncogene 36 (2017) 3609-3617, https://doi.org/10.1038/ onc. 2016.520.

[46] K. Tang, Y. Yu, L. Zhu, P. Xu, J. Chen, J. Ma, H. Zhang, H. Fang, W. Sun, L. Zhou, et al., Hypoxia-reprogrammed tricarboxylic acid cycle promotes the growth of human breast tumorigenic cells, Oncogene 38 (2019) 6970-6984, https://doi.org/ 10.1038/s41388-019-0932-1.

[47] M.X. Liu, L. Jin, S.J. Sun, P. Liu, X. Feng, Z.L. Cheng, W.R. Liu, K.L. Guan, Y.H. Shi, H.X. Yuan, et al., Metabolic reprogramming by PCK1 promotes TCA cataplerosis, oxidative stress and apoptosis in liver cancer cells and suppresses hepatocellular carcinoma, Oncogene 37 (2018) 1637-1653, https://doi.org/10.1038/s41388017-0070-6.

[48] E.D. Montal, R. Dewi, K. Bhalla, L. Ou, B.J. Hwang, A.E. Ropell, C. Gordon, W. J. Liu, R.J. DeBerardinis, J. Sudderth, et al., PEPCK coordinates the regulation of central carbon metabolism to promote cancer cell growth, Mol. Cell 60 (2015) 571-583, https://doi.org/10.1016/j.molcel.2015.09.025.

[49] E.D. Montal, K. Bhalla, R.E. Dewi, C.F. Ruiz, J.A. Haley, A.E. Ropell, C. Gordon, J. D. Haley, G.D. Girnun, Inhibition of phosphoenolpyruvate carboxykinase blocks lactate utilization and impairs tumor growth in colorectal cancer, Cancer, Metab 7 (2019) 8, https://doi.org/10.1186/s40170-019-0199-6, 019-0199-6. eCollection 2019.

[50] S.C. Burgess, N. Hausler, M. Merritt, F.M. Jeffrey, C. Storey, A. Milde, S. Koshy, J. Lindner, M.A. Magnuson, C.R. Malloy, et al., Impaired tricarboxylic acid cycle activity in mouse livers lacking cytosolic phosphoenolpyruvate carboxykinase, J. Biol. Chem. 279 (2004) 48941-48949, https://doi.org/10.1074/jbc. M407120200.

[51] A. Potts, A. Uchida, S. Deja, E.D. Berglund, B. Kucejova, J.A.G. Duarte, X. Fu, J. D. Browning, M.A. Magnuson, S.C. Burgess, Cytosolic phosphoenolpyruvate carboxykinase as a cataplerotic pathway in the small intestine, Am. J. Physiol. Gastrointest. Liver Physiol. 315 (2018) G249-G258, https://doi.org/10.1152/ ajpgi.00039.2018.

[52] P. She, M. Shiota, K.D. Shelton, R. Chalkley, C. Postic, M.A. Magnuson, Phosphoenolpyruvate carboxykinase is necessary for the integration of hepatic energy metabolism, Mol. Cell Biol. 20 (2000) 6508-6517, https://doi.org/ 10.1128/mcb.20.17.6508-6517.2000.

[53] S. Satapati, B. Kucejova, J.A. Duarte, J.A. Fletcher, L. Reynolds, N.E. Sunny, T. He, L.A. Nair, K.A. Livingston, X. Fu, et al., Mitochondrial metabolism mediates oxidative stress and inflammation in fatty liver, J. Clin. Invest. 125 (2015) 4447-4462, https://doi.org/10.1172/JCI82204.

[54] J.G. Ren, P. Seth, C.B. Clish, P.K. Lorkiewicz, R.M. Higashi, A.N. Lane, T.W. Fan, V. P. Sukhatme, Knockdown of malic enzyme 2 suppresses lung tumor growth, induces differentiation and impacts PI3K/AKT signaling, Sci. Rep. 4 (2014) 5414, https://doi.org/10.1038/srep05414.

[55] N. Jelluma, X. Yang, D. Stokoe, G.I. Evan, T.B. Dansen, D.A. Haas-Kogan, Glucose withdrawal induces oxidative stress followed by apoptosis in glioblastoma cells but not in normal human astrocytes, Mol. Cancer. Res. 4 (2006) 319-330, https://doi. org/10.1158/1541-7786.MCR-05-0061.

[56] S. Owada, Y. Shimoda, K. Tsuchihara, H. Esumi, Critical role of $\mathrm{H} 2 \mathrm{O} 2$ generated by NOX4 during cellular response under glucose deprivation, PloS One 8 (2013), e56628, https://doi.org/10.1371/journal.pone.0056628.

[57] C.J. De Saedeleer, P.E. Porporato, T. Copetti, J. Pérez-Escuredo, V.L. Payen, L. Brisson, O. Feron, P. Sonveaux, Glucose deprivation increases monocarboxylate transporter 1 (MCT1) expression and MCT1-dependent tumor cell migration, Oncogene 33 (2014) 4060-4068, https://doi.org/10.1038/onc.2013.454.

[58] B.G. Allen, S.K. Bhatia, J.M. Buatti, K.E. Brandt, K.E. Lindholm, A.M. Button, L. I. Szweda, B.J. Smith, D.R. Spitz, M.A. Fath, Ketogenic diets enhance oxidative stress and radio-chemo-therapy responses in lung cancer xenografts, Clin. Cancer Res. 19 (2013) 3905-3913, https://doi.org/10.1158/1078-0432.CCR-12-0287.

[59] G. Pani, R. Colavitti, B. Bedogni, R. Anzevino, S. Borrello, T. Galeotti, Determination of intracellular reactive oxygen species as function of cell density, Methods Enzymol. 352 (2002) 91-100, https://doi.org/10.1016/s0076-6879(02) 52010-3.

[60] V.E. Kagan, G. Mao, F. Qu, J.P. Angeli, S. Doll, C.S. Croix, H.H. Dar, B. Liu, V. A. Tyurin, V.B. Ritov, et al., Oxidized arachidonic and adrenic PEs navigate cells to ferroptosis, Nat. Chem. Biol. 13 (2017) 81-90, https://doi.org/10.1038/ nchembio. 2238 . 